\title{
Time-Resolved Rayleigh Scattering Measurements in Hot Gas Flows
}

\author{
Amy F. Mielke* \\ NASA Glenn Research Center, Cleveland, Ohio, 44135 \\ Kristie A. Elam ${ }^{\dagger}$ \\ Jacobs Sverdrup, Cleveland, Ohio, 44135 \\ and \\ Chih-Jen Sung ${ }^{\ddagger}$ \\ Case Western Reserve University, Cleveland, Ohio, 44106
}

\begin{abstract}
[Abstract] A molecular Rayleigh scattering technique is developed to measure timeresolved gas velocity, temperature, and density in unseeded gas flows at sampling rates up to $32 \mathrm{kHz}$. A high power continuous-wave laser beam is focused at a point in an air flow field and Rayleigh scattered light is collected and fiber-optically transmitted to the spectral analysis and detection equipment. The spectrum of the light, which contains information about the temperature and velocity of the flow, is analyzed using a Fabry-Perot interferometer. Photomultipler tubes operated in the photon counting mode allow high frequency sampling of the circular interference pattern to provide time-resolved flow property measurements. Mean and rms velocity and temperature fluctuation measurements in both an electrically-heated jet facility with a 10-mm diameter nozzle and also in a hydrogen-combustor heated jet facility with a $50.8-\mathrm{mm}$ diameter nozzle at NASA Glenn Research Center are presented.
\end{abstract}

\footnotetext{
${ }^{*}$ Research Engineer, Optical Instrumentation and NDE Branch, 21000 Brookpark Rd/M.S. 77-1, AIAA Member ${ }^{\dagger}$ Optics Technician

${ }^{\ddagger}$ Professor, Department of Mechanical and Aerospace Engineering, AIAA Associate Fellow
} 


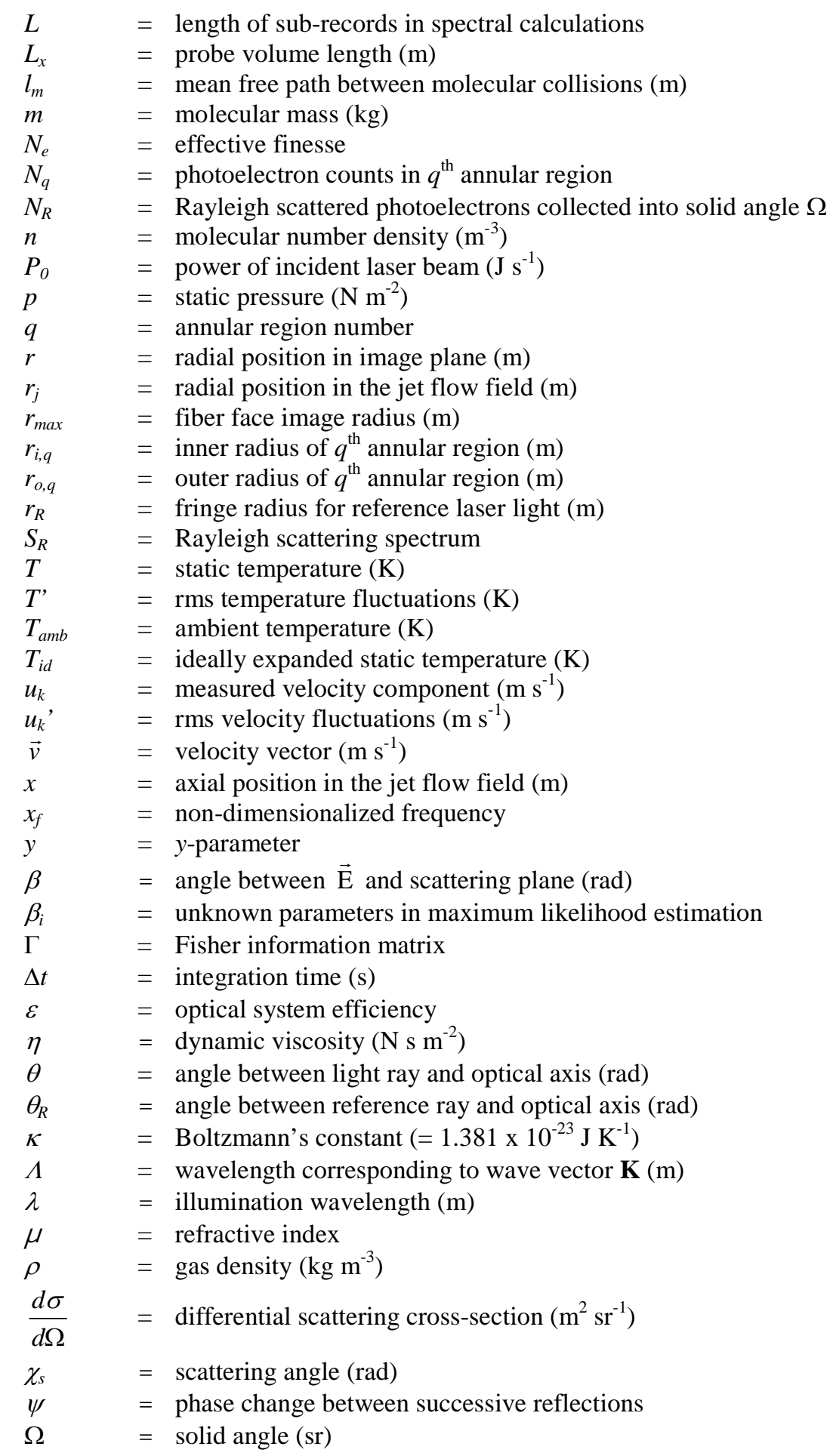

\section{Introduction}

CURRENTLY, non-intrusive temperature measurement techniques available for use in turbulent flow studies where the local pressure is unknown are limited to sampling rates less than a few $\mathrm{kHz}$. There are also no techniques that can provide non-intrusive time-resolved measurement of gas temperature, velocity, and density simultaneously at rates beyond a few $\mathrm{kHz}$. Conventional intrusive measurement devices such as resistance wires, pressure probes, and thermocouples are usually limited in spatial and temporal resolution, disturb the flow under study, and can be damaged by high pressure or temperature. The Rayleigh scattering technique presented is unique 
in that it provides simultaneous temperature, velocity, and density measurements at sampling rates up to $32 \mathrm{kHz}$. A high power single-frequency continuous-wave (cw) laser beam is focused at a point in an air flow field and Rayleigh scattered light is collected and transmitted via optical fiber to another location where the light is spectrally resolved. The signal strength and spectrum of the light contain information about the density, temperature, and velocity of the flow. A planar mirror Fabry-Perot interferometer (FPI) is used to analyze the spectrum of the scattered light, and photomultiplier tubes (PMTs) record the signal strength and fringe intensity pattern at high sampling rates enabling time-resolved measurement of the gas flow properties. The ability to obtain dynamic high frequency response measurements of multiple properties simultaneously is a valuable tool for compressible, turbulent flow research. Dynamic flow measurements are particularly useful to aeroacoustics researchers who are interested in correlating flow property fluctuations with far field acoustic fluctuations, leading to validation and improvement of computational aeroacoustics (CAA) noise prediction codes. Knowledge of velocity-temperature fluctuation correlations and turbulent Prandtl number are also needed to improve computational fluid dynamic (CFD) models of compressible turbulent flows. The ability to route the signal, and potentially the incident illumination, via fiber optics makes this technique appealing to facilities researchers who typically require flow measurements in harsh environments with tight space constraints.

Various optical techniques are available for temperature, molecular number density, and velocity measurements. Optical flow measurement techniques can be divided into two basic categories: 1. Molecular scattering techniques that involve either elastic (non-energy-exchanging) or inelastic (energy-exchanging) scattering processes from atoms or molecules, and 2. Particle scattering techniques that involve elastic scattering from particles (Mie scattering) entrained in the flow. Molecular-based techniques are free of problems associated with the seeding of flows with particulates, such as abrasiveness to equipment and difficulties with achieving proper flow seeding conditions, experienced in particle-based techniques. Since the gas molecule properties are directly determined, molecularbased techniques are capable of providing gas temperature and density information not available in particle-based techniques. Eckbreth ${ }^{1}$ provides the details of several molecular scattering based techniques, such as Coherent AntiStokes Raman Spectroscopy (CARS), spontaneous Raman and Rayleigh scattering, and Laser Induced Fluorescence (LIF), with an emphasis on their use in combustion applications. Other molecular-based techniques include laserinduced gratings ${ }^{2,3}$, filtered Rayleigh scattering ${ }^{4}$, collective light scattering ${ }^{5}$, and molecular flow tagging techniques ${ }^{6}$. The most common particle-based techniques are Laser Doppler Velocimetry (LDV) ${ }^{7}$, Particle Image Velocimetry $(\mathrm{PIV})^{8}$, and Planar Doppler Velocimetry (PDV) ${ }^{9}$.

The temporal response of most optical measurement techniques is typically limited by the repetition rate of pulsed lasers; usually on the order of 10-100 Hz. One can potentially take many snap shots to obtain rms turbulence measurements, but the snapshots must be acquired at repetition rates greater than most standard pulsed lasers in order to provide power spectral content. Advances in laser and camera technology have led to recent developments of temporally resolved measurements with pulsed lasers at rates exceeding $10 \mathrm{kHz}$. Although high repetition rate lasers exist, they do not have the narrow linewidth, stable frequency, and high beam quality typically needed in spectroscopic Rayleigh scattering flow diagnostics. Using a cw laser has many advantages over a pulsed-laser for obtaining quasi-instantaneous time-resolved Rayleigh scattering measurements. CW lasers have stable output power eliminating the need to sample the input beam to account for shot-to-shot pulse energy variations as with pulsed lasers. Collecting Rayleigh scattered light over a finite length of a high power cw laser beam, spectrally filtering the light, and sampling the number of photons collected during very short integration periods provides point-wise multiproperty measurements with spatial resolution of approximately $0.1 \times 0.1 \times 1 \mathrm{~mm}^{3}$ at high temporal resolution without the need for multiple lasers and complex alignment requirements.

Previous works using molecular Rayleigh scattering to make temperature, velocity, and number density measurements in various environments ${ }^{10-13}$, and dynamic density and velocity measurements in supersonic free jets $^{14,15}$ have been reported. The technique described in this paper was previously validated in an acoustically modulated nozzle flow and an asymmetric oscillating counterflow with unequal enthalpies; these modulated flows were used to validate the velocity and temperature fluctuation measurements, respectively, by comparison with hotwire and coldwire probe measurements ${ }^{16}$. The current work describes the application of the technique for characterization of an air flow issuing from an electrically-heated jet equipped with a 10-mm diameter nozzle that provides flow velocities up to $110 \mathrm{~m} / \mathrm{s}$ and temperatures up to $775 \mathrm{~K}$. The Rayleigh scattering measurements are compared with hotwire and coldwire measurements. In response to NASA's mission to reduce aircraft noise levels, experiments were recently conducted to evaluate the contribution of temperature fluctuations to far-field noise by application of the presented Rayleigh scattering technique in the Small Hot Jet Acoustic Rig (SHJAR), which is a heated nozzle facility located in the AeroAcoustic Propulsion Laboratory (AAPL) at NASA Glenn Research Center (GRC). This facility can provide up to Mach 2 flow conditions with maximum temperatures approaching $970 \mathrm{~K}$. Sound pressure fluctuation (microphone) measurements will eventually be acquired simultaneously with Rayleigh 
measurements allowing correlation between flow property fluctuations and noise generation. Determining sources of jet noise will help engineers to design quieter, more efficient aircraft. Data acquired in this facility at subsonic heated flow conditions using a 50.8-mm diameter convergent nozzle are presented. While all three flow parameters (density, temperature, and velocity) were measured in these two experiments, only velocity and temperature results are presented here since the density measurement is trivial and has been demonstrated in previous work ${ }^{15,17}$.

\section{Theory}

\section{A. Rayleigh scattering and spectral analysis}

In molecular Rayleigh scattering an incident electric field interacts with an atom or molecule inducing a dipole moment that oscillates and radiates at the frequency of the incident field. It is considered an elastic scattering process because the internal energy of the molecule is unchanged and the frequency of the light is changed only by the Doppler effect due to the molecular motion. When light from a single frequency laser beam passes through a gas, the scattered light is shifted in frequency by the Doppler effect due to the thermal as well as the bulk motion of the molecules. The frequency spectrum of the scattered light contains information about the gas density, bulk velocity, and temperature. Figure 1 shows a Rayleigh scattering spectrum containing the narrow laser line and a typical Rayleigh spectral peak to illustrate how the flow property measurements are obtained from the spectral information. If the gas composition is fixed, the total intensity of the Rayleigh scattered light is directly proportional to the gas density. The frequency shift between the laser peak and the Rayleigh peak is proportional to the bulk flow velocity. The width of the spectrum is related to the gas temperature.

The shape of the spectrum is dependent on gas pressure $p$ and temperature $T$, and the scattering angle $\chi_{\mathrm{s}}^{18}$. A non-dimensional parameter $y$, which represents the ratio of the wavelength of the scattering grating $\left(\Lambda=\frac{\lambda}{2 \sin \frac{\chi_{s}}{2}}\right)$ to the mean free path between molecular collisions $\left(l_{m}=\frac{a \eta}{{ }_{n \kappa T}}\right)$, is used to establish spectral shape regimes:

$$
y=\frac{\Lambda}{2 \pi l_{m}}=\frac{p}{\eta K a}
$$

where $a$ is the most probable molecular speed:

$$
a=\sqrt{\frac{2 \kappa T}{m}}
$$

$\lambda$ is the illumination wavelength, $\eta$ is the dynamic viscosity of the gas, $n$ is the molecular number density, and $\kappa$ is Boltzmann's constant. The interaction wave vector $\vec{K}$, which defines the direction of the velocity component being measured, is the bisector of the incident and scattered light wave vectors, $\overrightarrow{k_{0}}$ and $\overrightarrow{k_{s}}$, respectively (Fig. 2). The interaction wave vector and its magnitude $K$ are given by:

$$
\begin{aligned}
& \vec{K}=\overrightarrow{k_{s}}-\overrightarrow{k_{0}} \\
& K=|\vec{K}|=\frac{4 \pi}{\lambda}\left[\sin \frac{\chi_{s}}{2}\right]
\end{aligned}
$$

The geometry of the optical arrangement in an experiment can be designed such that the desired component of the velocity $u_{k}$ is measured:

$u_{k}=\frac{\vec{K} \cdot \vec{v}}{K}$

Experiments are typically arranged such that the electric field vector $\vec{E}$ is perpendicular to the scattering plane defined by the incident and scattered light wave vectors $\left(\beta=90^{\circ}\right.$, 's'-type polarization (Fig. 2)). In this situation the scattering is independent of the scattering angle. In the opposite situation where $\beta=0^{\circ}$ and $\vec{E}$ is in the scattering 


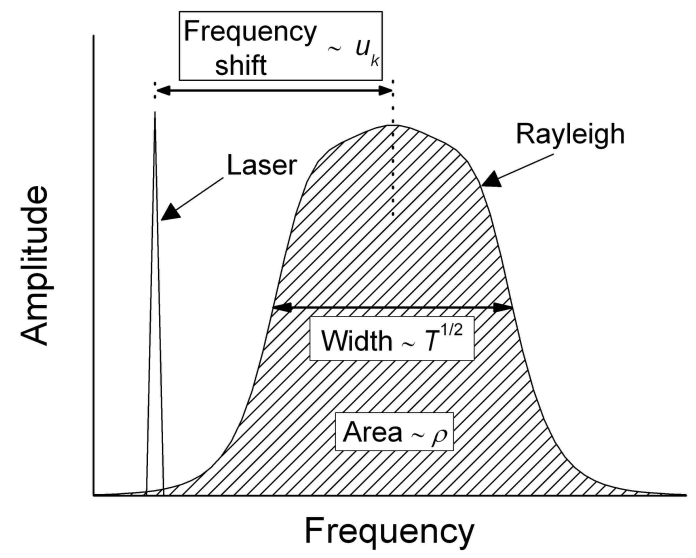

Figure 1. Rayleigh scattering spectrum.

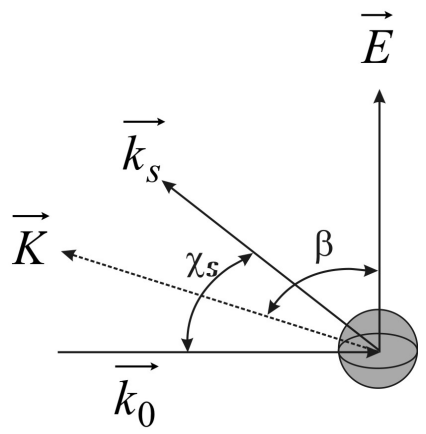

Figure 2. Light scattering from a moving particle.

plane ('p'-type polarization) the scattering is dependent on scattering angle and approaches zero as $\chi_{s}$ approaches $90^{\circ}$.

Three spectral shape regimes are defined for typical $\chi_{\mathrm{s}}=90^{\circ}$ scattering ${ }^{18-20}$. The shape regimes are illustrated in Fig. 3, where the normalized frequency is defined as:

$x_{f}=\frac{2 \pi\left(f-f_{0}\right)}{K a}$

where $f_{0}$ is the frequency of the incident laser light. For low density gases where $y<1$ the probability of molecular interactions is small and the molecular motion is thermally dominated. In this case the Rayleigh spectrum is determined only by the molecular velocity distribution which is accurately modeled by a Gaussian function for a Maxwellian velocity distribution. The gas is said to be in the Knudsen or collisionless regime. For higher density gases where $y>1$ and molecular motions become correlated, collective effects become significant. The Rayleigh spectrum broadens and eventually develops sidebands and the line shapes become Lorentzian. These sidebands are caused by thermally excited acoustic waves that cause density fluctuations to move at the speed of sound and are referred to as Brillouin-Mandel'shtam scattering ${ }^{21}$. This case is referred to as the hydrodynamic or continuum regime where molecular collisions are the dominant process ${ }^{22}$. Finally, for $0.2 \leq y \leq 2$, as is the case in the present experiments, the gas is in a transition region between the collisionless and hydrodynamic regimes, and a kinetic theory model is required. The line shape in this case is governed by both acoustic and thermal processes.

A kinetic theory model developed by Professor G. Tenti (TENTI S6: 6-moment Rayleigh scattering model)

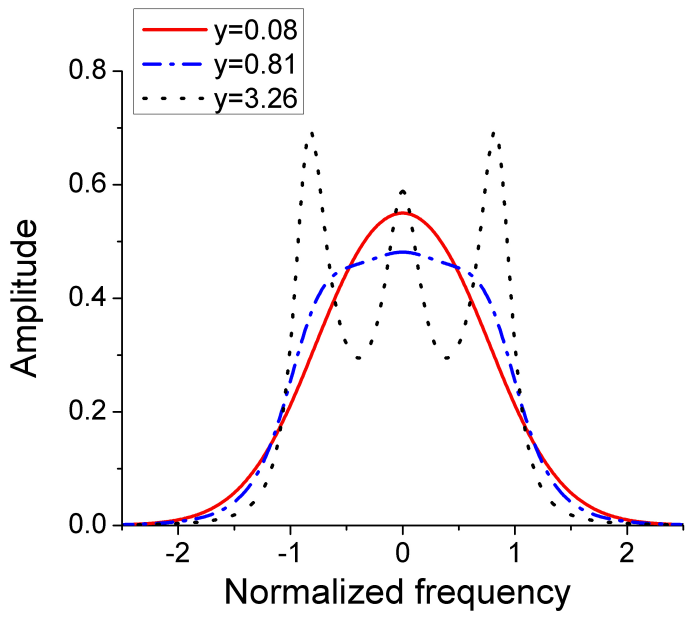

Figure 3. Rayleigh scattering spectrum for various $y$-parameters. provides the Rayleigh-Brillouin spectrum for diatomic gases in all density regimes ${ }^{18,23}$. This is the most accurate model currently available, although it is not exact since it assumes a single species. The TENTI S6 model requires values for shear viscosity, thermal conductivity, molecular weight, internal specific heat, and bulk viscosity of the gas in addition to the $y$-parameter in order to generate the 


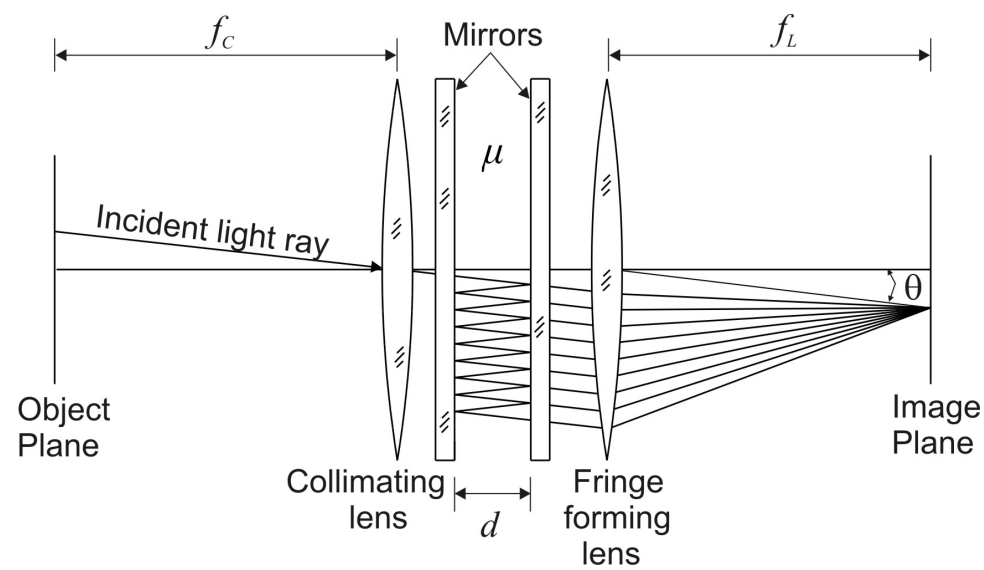

Figure 4. Fabry-Perot interferometer

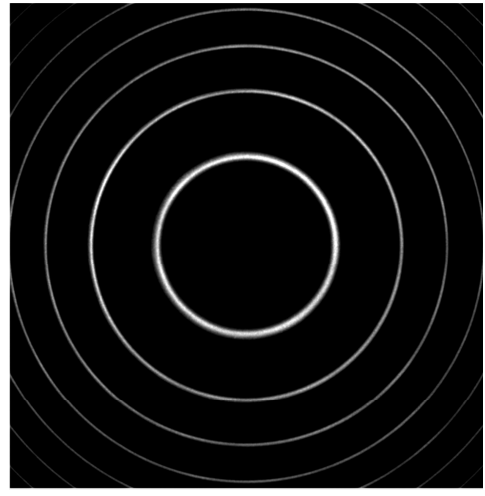

Figure 5. Concentric ring interference pattern from a planar single frequency light source imaged through a Fabry-Perot interferometer operated in the static imaging mode.

spectral information. The shear viscosity, thermal conductivity, and mean molecular weight for air, and nitrogen values of internal specific heat and bulk viscosity (as air values were not available) were used for generation of the Rayleigh spectrum using the TENTI S6 model calculated using a computer code provided by Professor G. Tenti. This spectrum model was used to generate the information displayed in Figs. 1 and 3, and is incorporated in the model function used in maximum likelihood estimation (MLE) analysis of the experimental data.

The spectrum of the Rayleigh scattered light is analyzed using a planar mirror FPI (Fig. 4) ${ }^{24}$. The Fabry Perot etalon consists of two parallel planar mirrors with their reflective surfaces facing each other. Maximum transmission occurs when the optical path-length difference $(O P D)$ between each transmitted beam is equal to an integer multiple of the wavelength, or

$$
O P D=2 \mu d \cos \theta=m_{i} \lambda,
$$

where $\mu$ is the index of refraction of the medium in the cavity between the mirrors, $d$ is the mirror spacing, $\theta$ is the angle between the incoming light ray and the optical axis, and $m_{i}$ are integer values representing the order of interference. In the static "imaging mode" the plate spacing and medium refractive index are held constant while the angle of the incoming light ray $\theta$ is varied by imaging points off-axis. A lens at the etalon output images the light source at the image plane where a detector is located. A planar source emitting monochromatic light imaged through the etalon results in an image of the source modulated in intensity by concentric light rings at locations associated with angles that meet the criterion of Eq. (7) as shown in Fig. 5. If the incoming light has a very narrow linewidth the imaged intensity pattern will essentially be a delta function convolved with the Airy function. For spectrally broadened light, such as Rayleigh scattered light, the rings will broaden accordingly; the linewidth of the rings provides a measure of temperature from Rayleigh scattered light. The frequency shift of the light associated with the bulk flow velocity can be determined by a spatial shift in the ring positions in the image. An alternative mode of operation of the Fabry-Perot is the "scanning mode" which involves varying either the mirror spacing $d$ or the index of refraction $\mu$ of the medium in the cavity to allow transmission of different frequencies to occur and recreate the Rayleigh spectrum. However, this mode can only be used for time-averaged measurements since a finite length of time is required to perform the "scanning". The imaging mode allows instantaneous and time-resolved measurements to be obtained since $\theta$, and hence the spectrum, is scanned instantaneously.

In this work the FPI is operated in the static imaging mode. The fringe intensity pattern is a function of both the Rayleigh spectrum and the Fabry-Perot instrument function. The Fabry-Perot instrument function as a function of normalized frequency $x_{f}$ and radial position in the image plane $r$ is given by the following relation:

$$
I_{F P}\left(x_{f}, r\right)=\frac{1}{1+F \sin ^{2} \frac{\psi\left(x_{f}, r\right)}{2}}
$$

where $F$ is the instrument function contrast defined as: 


$$
F=\frac{1}{\sin ^{2}\left(\frac{\pi}{2 N_{e}}\right)}
$$

and $\psi$ is the phase change between successive reflections:

$$
\psi=\frac{4 \pi \mu d}{\lambda}\left[\frac{\theta_{R}^{2}-\theta^{2}}{2}+\frac{\lambda a}{c \Lambda} x_{f}\right]
$$

where $\theta=r / f_{L}$

$f_{L}$ is the fringe forming lens focal length, and $\theta_{R}$ is the angle between the optical axis and the light ray associated with the incident laser frequency.

Rayleigh scattered light from a defined probe volume is collected into a multimode optical fiber. In this experiment the incident laser beam with power $P_{0}$ is focused to a diameter smaller than the imaged field size, such that the power collected is proportional to the length of the probe volume $L_{x}$ set by the field size of the collection optics. The Rayleigh scattered power scattered into solid angle $\Omega$ from a probe volume of length $L_{x}$ expressed in terms of expected photoelectron counts $\left\langle N_{R}\right\rangle$ is:

$$
\left\langle N_{R}\right\rangle=\frac{\varepsilon P_{0} n L_{x} \lambda \Omega \Delta t}{h c}\left(\frac{d \sigma}{d \Omega}\right) \sin ^{2} \beta
$$

where the overall system efficiency $\varepsilon$ includes detector quantum efficiency and other losses, $\Delta t$ is the integration time over which photoelectrons are counted, $h$ is Planck's constant, and $\frac{d \sigma}{d \Omega}$ is the differential scattering cross-section. The scattering cross-section at the laser wavelength of $532 \mathrm{~nm}$ was obtained using published refractive index data for air as $5.9 \times 10^{-32} \mathrm{~m}^{2} / \mathrm{sr}$.

The light exiting the fiber is collimated and directed to the FPI, and a lens at the interferometer output focuses the interference fringe pattern at the image plane. With the interferometer in the light path, the interference fringe pattern is equivalent to the intensity of the image of the fiber face without the Fabry-Perot modified by the transmission properties of the FPI. The circular interference pattern is split up into one circular and three annular regions as shown in Fig. 6, and the light from each region is detected by four PMT's. The expected amount of energy collected from the $q^{\text {th }}$ annular region of the interference pattern in terms of photoelectron counts, can be expressed by the following model function:

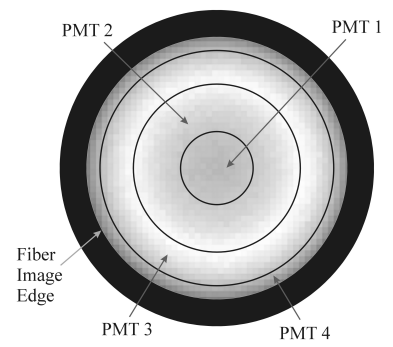

Figure 6. Dissection of FabryPerot fringe pattern into one circular and three annular regions.

$$
\left\langle N_{q}\right\rangle=\frac{\left\langle N_{R}\right\rangle}{\pi r_{\max }^{2}} 2 \pi \int_{r_{i, q}}^{r_{o, q}} \int_{-\infty}^{\infty} S_{R}\left(x_{f}\right) I_{F P}\left(x_{f}, r\right) r d x_{f} d r
$$

where $r_{\max }$ is the radius of image of the fiber face, $r_{i, q}$ and $r_{o, q}$ are the inner and outer radii of the $q^{\text {th }}$ annular region, respectively, and the Rayleigh spectrum $S_{R}$ is evaluated using the TENTI S6 model. The developed model function given by Eq. (13) is used to evaluate the lower bound measurement uncertainty, which will be presented in section II.B., and is used as the model function in maximum likelihood estimation (MLE) analysis of the experimental data.

The Fabry-Perot used in the experiments has a free spectral range (FSR), or frequency change between fringes, of $8.7 \mathrm{GHz}$ and effective finesse of approximately 15 , which provides an instrument function with a width of 0.58 $\mathrm{GHz}$. The width of the Rayleigh spectrum is set by the molecular thermal motion. For a temperature of $300 \mathrm{~K}$ the Rayleigh spectral linewidth is approximately $1.1 \mathrm{GHz}$; hence the resolution of the interferometer is sufficient to resolve the Rayleigh spectrum. The imaged fringe pattern is limited by the finite diameter of the optical fiber face that is imaged through the Fabry-Perot. The image diameter in the current experiments is equivalent to a partial fringe order and therefore, only the innermost fringe of the pattern is detectable, as shown in Fig. 6. The outer diameter of the image is equivalent to approximately 0.4 times the FSR of the instrument, which is equivalent to a maximum frequency shift of approximately $3.5 \mathrm{GHz}$. This means that the maximum velocity that can be measured 
must result in a frequency shift of less than this amount. The velocity associated with a $3.5 \mathrm{GHz}$ frequency shift for the current experimental arrangement is $1300 \mathrm{~m} / \mathrm{s}$. This would require a reference fringe that is right at the edge of the image and a Doppler shifted fringe at the origin of the concentric pattern. In reality, this would be an impossible situation to measure. A more realistic velocity measurement limit would be approximately half of this or $650 \mathrm{~m} / \mathrm{s}$.

\section{B. Lower bound for measurement uncertainty}

The lower bound on the uncertainty in temperature, velocity, and density measurements using Rayleigh scattering is set by the photon statistical (shot) noise. Shot noise can be modeled by a Poisson distribution. Estimates of the measurement uncertainty in the unknown parameters for the Rayleigh measurement technique were obtained by Cramer-Rao lower bound analysis ${ }^{25}$. For a measurement that is a function of a set of unknown parameters $\beta_{i}=\left[\beta_{1}, \beta_{2}, \beta_{3}, \ldots\right]$ the variance $\sigma_{i}^{2}$ of the estimates $\hat{\beta}_{i}$ of the parameters is:

$$
\sigma_{i}^{2} \geq\left[\Gamma^{-1}\right]_{i i}
$$

where the Fisher information matrix for Poisson statistics is given by:

$$
\Gamma_{i, j}=\sum_{k} \frac{1}{\left\langle N_{k}\right\rangle} \frac{\partial\left\langle N_{k}\right\rangle}{\partial \beta_{i}} \frac{\partial\left\langle N_{k}\right\rangle}{\partial \beta_{j}}
$$

If a Gaussian spectrum is assumed, the lower bounds for uncertainty or standard deviation in the instantaneous temperature $T$, velocity $u_{k}$, and density $\rho$ measurements for an ideal instrument are:

$$
\sigma_{T} \geq\left(\frac{2 T^{2}}{\left\langle N_{R}\right\rangle}\right)^{1 / 2} ; \quad \sigma_{u_{k}} \geq\left(\frac{a^{2}}{2\left\langle N_{R}\right\rangle}\right)^{1 / 2} ; \quad \sigma_{\rho} \geq\left(\frac{\rho^{2}}{\left\langle N_{R}\right\rangle}\right)^{1 / 2}
$$

The lower bound uncertainty in temperature, velocity, and density estimates for air at a temperature of $298 \mathrm{~K}$ and standard atmospheric pressure for an ideal loss-free instrument that samples the data at a rate of $32 \mathrm{kHz}$ are as follows:

$$
\sigma_{T} \geq 1.65 \mathrm{~K} \quad \sigma_{u_{k}} \geq 1.14 \mathrm{~m} / \mathrm{s} \quad \sigma_{\rho} \geq 0.0046 \mathrm{~kg} / \mathrm{m}^{3}
$$

For any real instrument, these measurement uncertainties will be higher. Using the model function (Eq. 13) developed for $\left\langle N_{q}\right\rangle$, the lower bound measurement uncertainty in each parameter estimate was determined by calculation and inversion of the Fisher information matrix. The model function takes into account the FPI, quantum efficiency of the PMTs, and additional signal loss throughout the optical system due to coupling losses into the optical fiber and other transmission losses. The TENTI S6 spectral model was used to calculate the Rayleigh scattering spectrum in this analysis rather than using the simpler, less accurate Gaussian model used in the analysis above $^{18,23}$. The uncertainty analysis was performed for a sampling rate of $32 \mathrm{kHz}$, a probe volume length of $1.1-\mathrm{mm}$ and $f / 4$ collection optics, which correspond to the values for the electrically-heated jet experiment. The optical system installed in the SHJAR facility had slightly different characteristics; however the uncertainty estimates should be the same order of magnitude since both experiments resulted in similar photon count signal levels.

The Cramer-Rao lower bound analysis was used to optimize the experiment parameters, such as reference fringe radius $\left(r_{R}\right)$ and the radii of the fringe dissection mirrors. The parameters were varied in the model until the uncertainty levels in both $T$ and $u_{k}$ were minimized. Assuming a maximum fiber image diameter of $18.5 \mathrm{~mm}$, fringe dissection mirrors with radii of $2.5-\mathrm{mm}, 6-\mathrm{mm}, 8-\mathrm{mm}$ and $25-\mathrm{mm}$ were chosen for the concentric mirror system. The optimum reference fringe radius varies depending on the flow velocity; values between 6.5 - $\mathrm{mm}$ and 7.2 -mm were used in the experiments. Details of the optimization analysis can be found in Ref. 26. Once the optical system parameters were optimized, the Cramer-Rao lower bound was calculated over the expected range of flow conditions at a data acquisition rate of $32 \mathrm{kHz}$. Figure 7 shows the absolute uncertainty in the instantaneous temperature estimates for a static temperature range from $298 \mathrm{~K}$ to $773 \mathrm{~K}$ and a range of velocities from $-400 \mathrm{~m} / \mathrm{s}$ to $200 \mathrm{~m} / \mathrm{s}$, and Fig. 8 shows the absolute uncertainty in instantaneous velocity estimates for the same range of temperatures and velocities. The optical system parameters used in this analysis were optimized for the velocity range from $-120 \mathrm{~m} / \mathrm{s}$ to $0 \mathrm{~m} / \mathrm{s}$; hence, the region of the curves exhibiting minimum uncertainty occurs in this velocity range. The uncertainty increases outside of this velocity range since the shifted fringe is located either too close to the center of 
the image or too close to the outer edge of the image such that information is lost outside of the imaged region. Measurement uncertainty for $T$ and $u_{k}$ increases as temperature increases as shown in Figs. 7 and 8 . The uncertainty in both temperature and velocity is inversely related to the square-root of the number of photoelectron counts as indicated by Eq. (16). The number of photon counts decreases as temperature increases; this is attributable to the lower gas density at higher temperatures, resulting in fewer scattering molecules, and hence fewer scattered photons. Although the uncertainty in the parameters is rather high for instantaneous measurements, long data records allow for calculation of higher accuracy statistical quantities such as power spectra and mean square fluctuations.

A numerical simulation has been developed that uses the model function given by Eq. (13) to generate photon count data based on input velocity and temperature values. Ideal (noise-free) data were computed in terms of expected photon counts from each of the five PMTs. Photon count values were generated with Poisson noise and recorded in a data file. The details of the numerical simulation can be found in Ref. 26. Long data records of $32 \mathrm{kHz}$ data rate synthetic photon count data with Poisson statistics noise were generated assuming uniform non-turbulent velocity and temperature records, and optical system parameters similar to the electrically heated jet experiment. The noisy data was analyzed in the same way as experimental data using MLE to provide density, temperature, and velocity estimates. The mean temperature and velocity values were calculated from the processed data records and were subtracted from their respective time history records to provide zero-mean records, which were used to calculate the power spectral densities of the property fluctuations. The power spectra of the time records were calculated using a technique called the Welch method of modified periodograms, which provides an average of several shorter overlapped periodograms to minimize the variance in the spectral estimates ${ }^{27}$. The resulting power spectra provide fluctuation information out to $16 \mathrm{kHz}$ with $7.8125 \mathrm{~Hz}$ resolution, and the sum of all points in the spectra is equivalent to the mean square fluctuations. Figures 9 and 10 show the temperature and velocity power spectra calculated from the synthetic data for a steady mean temperature and velocity of $423 \mathrm{~K}$ and $-40 \mathrm{~m} / \mathrm{s}$, respectively. Since the actual data is steady (no fluctuations) in these simulation studies the power spectrum calculations provide insight into the uncertainty or noise levels in the calculated second order statistical values. The broadband shot noise causes a pedestal or offset in the power spectrum. This baseline noise floor provides the lower limit of measurable property fluctuations. The fluctuation baseline or lower limit was calculated using the spectral noise floor and frequency resolution of the power spectrum for the synthetic data. A summary of the temperature and velocity fluctuation amplitude lower bound calculated from the spectral noise floor values for the range of temperatures and velocities explored in these simulation cases are provided in Figs. 11 and 12, respectively. The uncertainty in the fluctuation estimates $\left(2^{\text {nd }}\right.$ order statistics) is significantly lower than the uncertainty in instantaneous parameter estimates. This conclusion is based on comparison of Fig. 7, which provides the rms error in instantaneous temperature estimates, and Fig. 11, which provides the lower bound of temperature fluctuation estimates, and likewise comparisons of Figs. 8 and 12 for instantaneous velocity and fluctuation estimates.

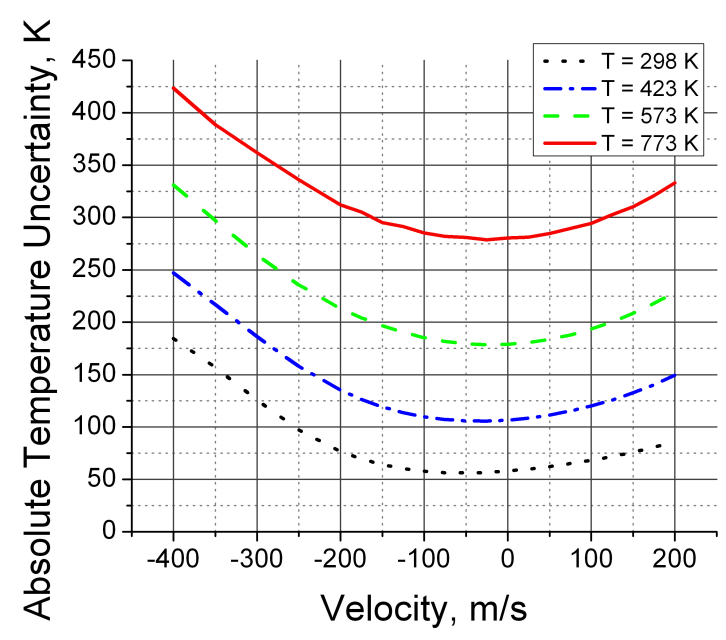

Figure 7. Absolute uncertainty for instantaneous temperature estimates for $32 \mathrm{kHz}$ sampling rate.

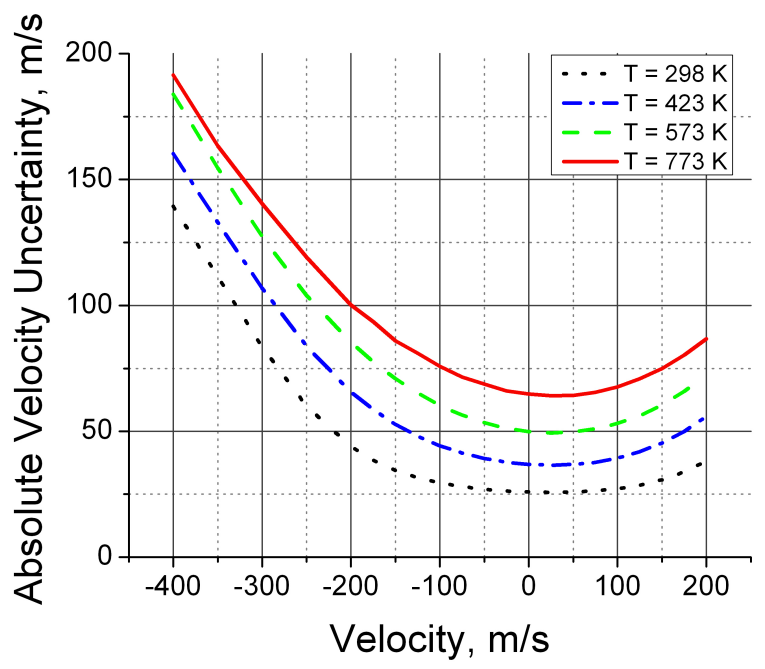

Figure 8. Absolute uncertainty for instantaneous velocity estimates for $32 \mathrm{kHz}$ sampling rate. 


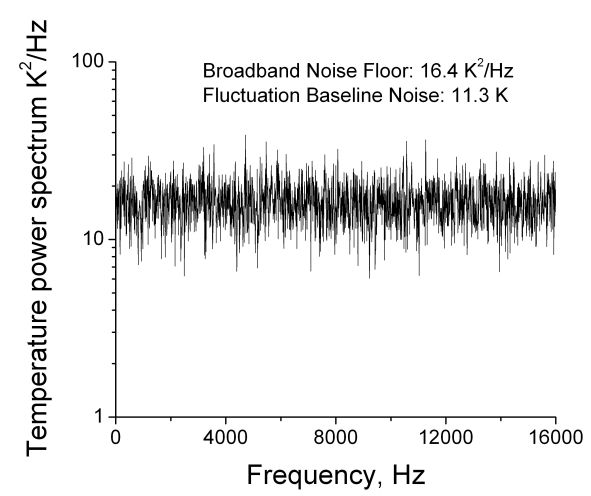

Figure 9. Temperature power spectrum calculated from synthetic data with steady temperature of $423 \mathrm{~K}$ and steady velocity of $-40 \mathrm{~m} / \mathrm{s}$.

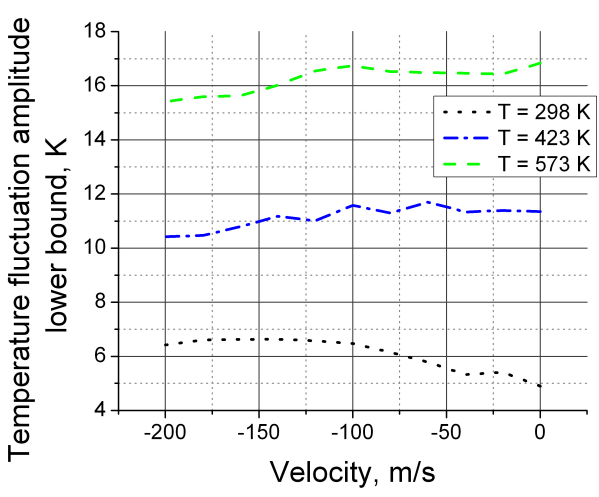

Figure 11. Temperature fluctuation amplitude lower bound based on spectral noise floor values.

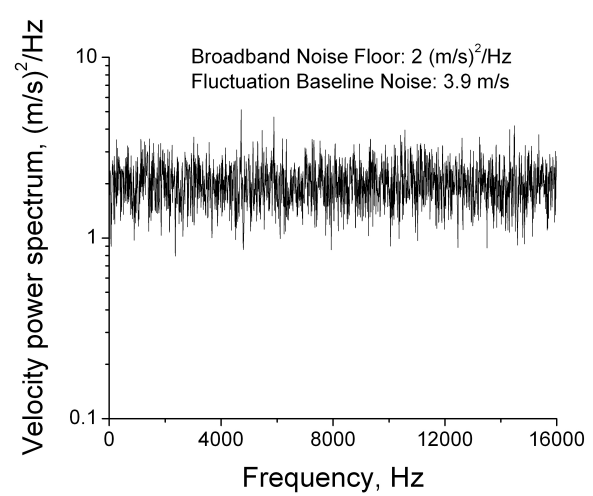

Figure 10. Velocity power spectrum calculated from synthetic data with steady temperature of $423 \mathrm{~K}$ and steady velocity of $-40 \mathrm{~m} / \mathrm{s}$.

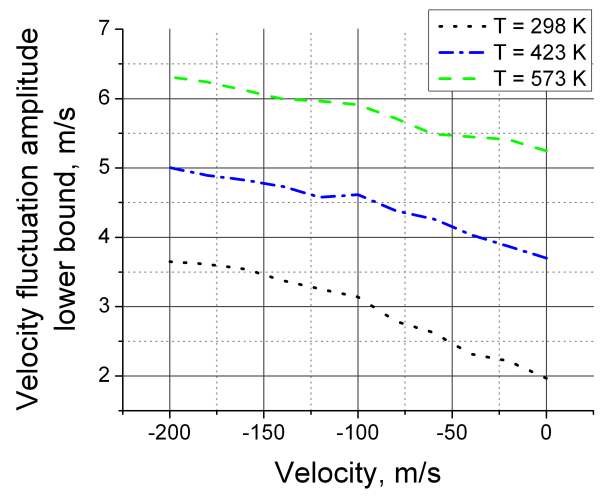

Figure 12. Velocity fluctuation amplitude lower bound based on spectral noise floor values.

\section{Experimental Setup}

The Rayleigh scattering diagnostic was applied in two heated jet facilities at NASA GRC. The first demonstration was in a lab-scale electrically-heated jet with a 10-mm diameter nozzle. The Rayleigh system was then installed in the Small Hot Jet Acoustic Rig (SHJAR) in NASA Glenn's AeroAcoustic Propulsion Laboratory. The SHJAR was equipped with a 50.8-mm diameter convergent nozzle and the flow was heated by a hydrogen combustor. The Rayleigh scattering system was used to measure temperature, axial velocity, and density fluctuations in both facilities. The goal of the testing in the SHJAR was to provide an experimental database, particularly of temperature fluctuation data, to be used for validation of computational codes.

\section{A. Electrically-Heated Nozzle Facility}

Flow measurements were acquired in an electrically heated air flow issuing from a $10-\mathrm{mm}$ exit diameter convergent nozzle with a $200-\mathrm{mm}$ diameter $10 \mathrm{~m} / \mathrm{s}$ filtered air co-flow (Fig. 13). Since the measurement technique relies on having particulate free gas flow, a series of filters were placed in line with the air plumbing to remove dust, oil and water from the air supply prior to entering the nozzle flow system. The air issuing from the jet was heated by applying voltage to coils of kanthal wires inside the jet plenum. The co-flow was provided by an air blower system equipped with submicron HEPA filters. Figure 13 shows the layout of the optics around the jet, which were used to collect Rayleigh scattered light from gas molecules in the flow. The jet was mounted such that the main flow direction was parallel to the table. A Coherent Verdi 10W, 532-nm wavelength, single-frequency, Nd:Vanadate $\mathrm{cw}$ laser provided incident light for the system. The laser beam was focused with a $250-\mathrm{mm}$ focal length lens (L1) to a $70-\mu \mathrm{m} 1 / \mathrm{e}^{2}$ diameter at the probe volume. The beam was oriented in the horizontal plane, at a $45^{\circ}$ angle to the primary flow direction. Light was collected at a $90^{\circ}$ scattering angle, collimated by a $f / 4200-\mathrm{mm}$ focal length lens (L2), and focused by a $100-\mathrm{mm}$ focal length lens (L3) onto a $0.55-\mathrm{mm}$ diameter multimode optical fiber. Since the 


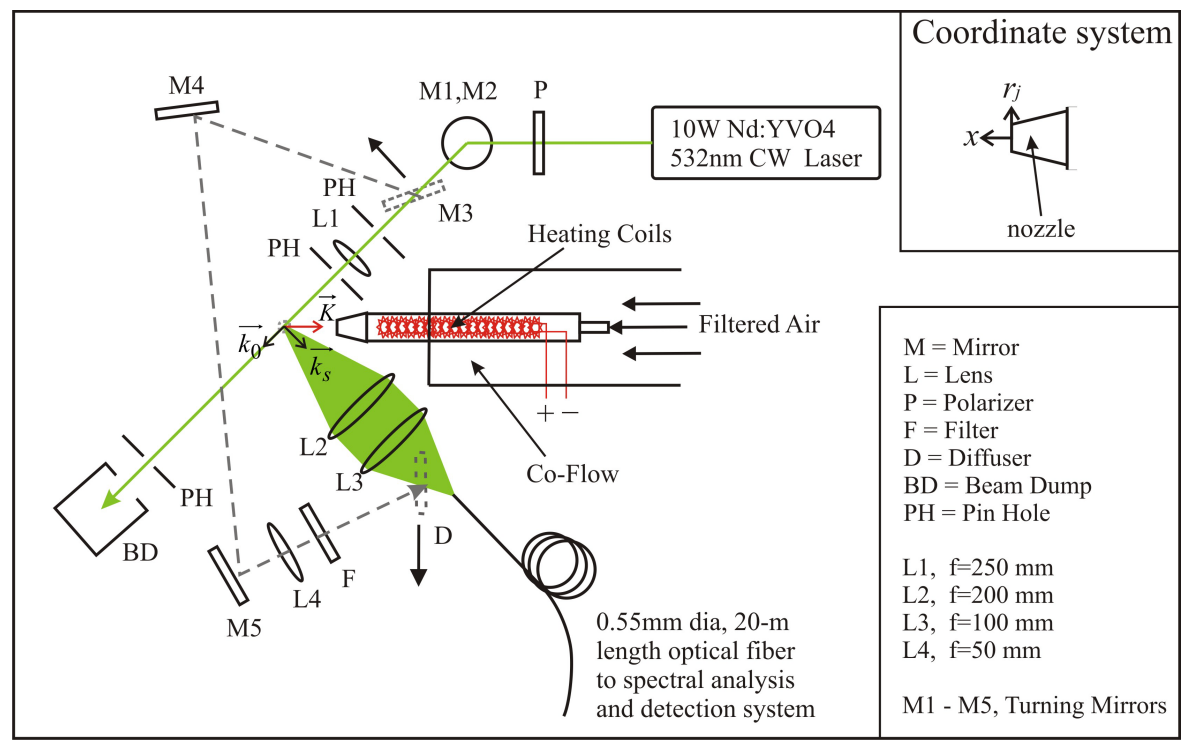

Figure 13. Diagram of laser and collection optics for acoustically-excited nozzle flow experiment

pair of lenses provided 1:2 imaging, the length of the probe volume in the laser beam propagation direction was 1.1$\mathrm{mm}$; however the resolution in the radial direction, which is the direction of the velocity gradient of interest, was $0.78-\mathrm{mm}$. The 20-m long optical fiber transmitted the scattered light to another area of the room where the spectral analysis equipment was located. The incident and scattering wave vectors were arranged such that the axial component of the jet velocity was measured, as indicated by the $\vec{K}$ vector (shown in red) in Fig. 13. The jet was mounted on vertical and horizontal translation stages so that the probe volume could be positioned anywhere in the jet plume. The probe volume was positioned two jet diameters from the nozzle exit and was scanned radially at 1$\mathrm{mm}$ increments across the flow providing velocity, density, and temperature profiles at various flow conditions. The jet was operated at a mean (steady flow) exit velocity that covered a range of approximately $10-110 \mathrm{~m} / \mathrm{s}$ and static temperature range of $295-775 \mathrm{~K}$, giving a Reynolds number range of $10^{3}-10^{5}$. The jet temperature was set by observing the output of a thermocouple in the jet plenum. A fixed power level was applied to the coils, which maintained a fixed air temperature for a fixed flow rate.

For calibration purposes, temperature, density, and velocity measurements were acquired in a steady laminar flow in the jet core. The mean static temperature at the probe volume location and the ambient air temperature were measured by 36 gauge wire $(0.13 \mathrm{~mm}$ bead diameter) type $\mathrm{K}$ thermocouples. Two digital pressure gauges measured the ambient (static) pressure and the jet plenum (total) pressure. The velocity and density at the probe location within the core flow were calculated via isentropic flow and ideal gas equations using total and static pressure and ambient temperature measurements. The calibration data was acquired in the core flow over a temperature range of $298 \mathrm{~K}$ to $765 \mathrm{~K}$ and a velocity range of $14 \mathrm{~m} / \mathrm{s}$ to $110 \mathrm{~m} / \mathrm{s}$. The velocity and temperature fluctuations were verified using hotwire anemometry and coldwire thermometry systems. The hotwire and coldwire systems could not be used at all flow conditions due to physical limitations of the devices. Both of the fine wire probes were limited to a maximum temperature of $423 \mathrm{~K}$. Also, the single wire hotwire system is not valid in flows with large temperature fluctuations, and the calibration must be performed at the flow temperature in which measurements are desired. Therefore, the hotwire measurements were performed in unheated flows with similar mean velocities as the corresponding heated cases. The coldwire probe broke when it was subjected to a flow velocity of $110 \mathrm{~m} / \mathrm{s}$; therefore coldwire data is only available at low speed flow conditions.

\section{B. Small Hot Jet Acoustic Rig (SHJAR)}

The SHJAR is a hot jet rig that can cover the range of Mach numbers up to Mach 2, and static temperature ratios up to 2.8 using a hydrogen combustor and central air compressor facilities. The SHJAR is located in the AeroAcoustic Propulsion Laboratory (AAPL) at NASA Glenn Research Center. The AAPL is a 19.8-m radius geodesic dome with its interior covered by sound absorbent wedges that provide the anechoic environment required to study propulsion noise from the SHJAR rig. The rig is positioned to exhaust out the open doorway, which eliminates background noise from flow collectors; however daytime sunlight imposed limitations on the use of extremely light sensitive optical instrumentation such that all testing was completed during twilight hours. The 


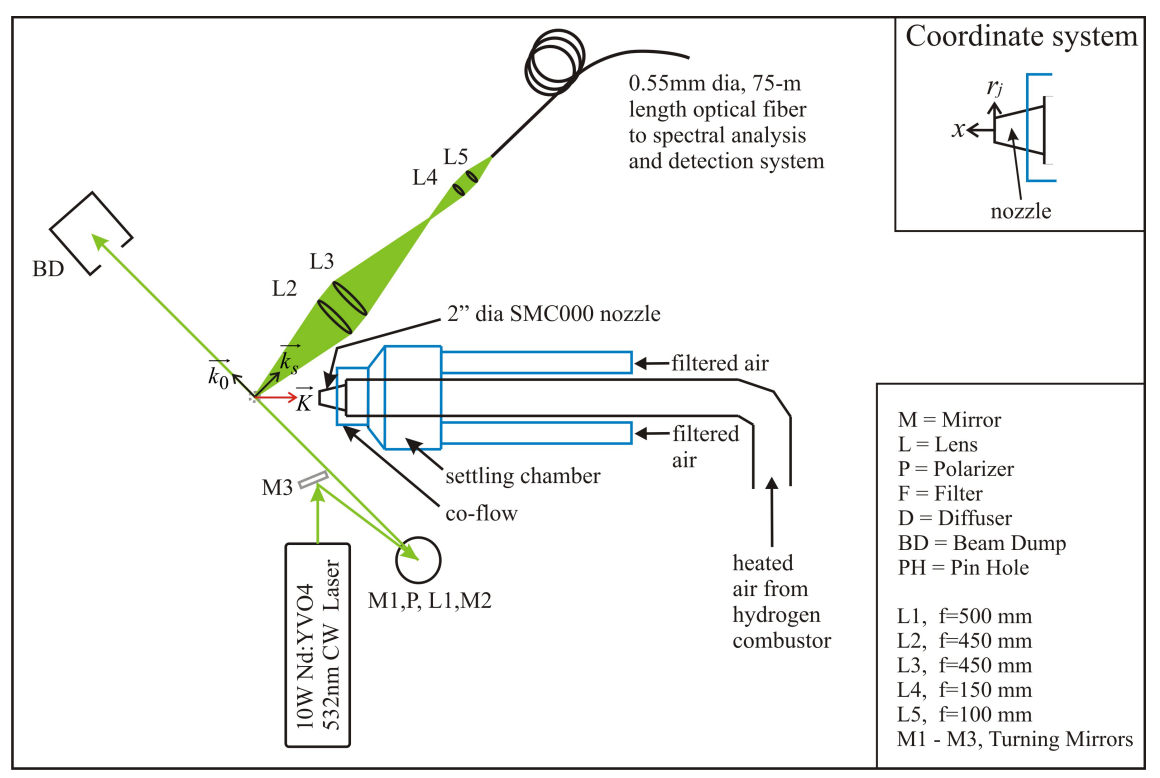

Figure 14. Diagram of laser and collection optics in the SHJAR facility.

nozzle used in this test is one of a family of convergent nozzles, called the Simple Metal Chevron (SMC) nozzles. The baseline nozzle (SMC000) was used in this test, which has a 50.8-mm exit diameter and has a smooth round exit (no chevrons). A variety of conditions with acoustic Mach number (Ma), which is defined as the ideally expanded velocity divided by the ambient sound speed, ranging from 0.5 to 1.59 and static temperature ratio (TR) from 0.835 to 2.7 were covered in the test matrix; however results from a single setpoint at $\mathrm{Ma}=0.5$ and $\mathrm{TR}=1.765$ are presented here. Measurements were acquired at several axial locations of $x / D \geq 2$; data will only be presented for a single axial location of $x / D=3$ and radial locations of $0.0 \leq r_{j} / D \leq 1.1$.

The laser and collection optics portion of the Rayleigh scattering system are shown around the SHJAR in Fig. 14. A co-flow system was built specifically for this test (components are shown in blue in Fig. 14) to provide a low speed particulate-free air stream around the jet core. Room air was filtered by a blower system equipped with submicron HEPA filters and was routed to the settling chamber via two 200-mm flex duct sections and exhausted around the nozzle through a straight $250-\mathrm{m}$ long section of $305-\mathrm{mm}$ diameter pipe. A screen was positioned between the settling chamber and the straight exhausting duct section to break up turbulence and straighten the flow. The top view of the facility shown in Fig. 14 illustrates the laser beam propagation and scattered light collection. A Coherent Verdi 10W, 532-nm wavelength cw laser provided incident light for the system. The laser head was located about 1$\mathrm{m}$ below the scattering plane and an enclosed box containing two turning mirrors, a polarizer, and the focusing lens routed the beam upwards (out of the plane of the page in Fig. 14) and through the centerline of the jet flow. The laser beam was focused with a 500-mm focal length lens (L1) to a $140-\mu \mathrm{m} 1 / \mathrm{e}^{2}$ diameter at the probe volume. The beam was oriented in the horizontal plane, at a $45^{\circ}$ angle to the primary flow direction. Light was collected at a $90^{\circ}$ scattering angle, collimated and focused by a pair of $f / 3450-\mathrm{mm}$ focal length lenses (L2 \& L3), then re-imaged by another pair of lenses having focal lengths of 150-mm and 100-mm (L4 \& L5, respectively) onto a 0.55-mm diameter multimode optical fiber. Since the train of lenses provided 1:1.5 imaging, the length of the probe volume in the laser beam propagation direction was $0.825-\mathrm{mm}$, providing resolution in the radial direction of $0.58-\mathrm{mm}$. The incident and scattering wave vectors were arranged such that the axial component of the jet velocity was measured, as indicated by the $\vec{K}$ vector (shown in red) in Fig. 14. The laser and beam propagation optics and the collection optics were all mounted on a large $\mathrm{x}-\mathrm{y}$ traversing system (not shown in the figure) so that the probe volume could be positioned at various locations in the jet flow. The $75-\mathrm{m}$ long optical fiber transmitted the scattered light to an adjacent building where the sensitive spectral analysis equipment was located.

For calibration purposes, temperature, density, and velocity measurements were acquired in a steady laminar flow in the jet core. The velocity, static temperature, and static density were calculated assuming ideally expanded flow. Calibration data was acquired in the core flow over a static temperature range of 239 to $630 \mathrm{~K}$ and a velocity range of 167 to $300 \mathrm{~m} / \mathrm{s}$. Data was then acquired at radial locations of $r_{j} / D=0,0.25,0.37,0.44,0.5,0.56,0.62,0.8$, and 1.1 at an axial location of $x / D=3$ for jet exit conditions of $500 \mathrm{~K}$ and $167 \mathrm{~m} / \mathrm{s}$. The optical system losses in this facility reduced the signal strength by about $1 / 2$ compared to the signals observed in the electrically-heated jet 
experiment due to additional collection and transmission losses; hence we were limited to a sampling rate of $10 \mathrm{kHz}$ for this test to achieve similar signal levels. Previous velocity data acquired using a hotwire probe at $10 \mathrm{kHz}$ data rate with a $4 \mathrm{kHz}$ anti-aliasing filter, and conventional PIV at $10 \mathrm{~Hz}$ data rate are shown for comparison.

\section{Spectral Analysis and Detection Optics}

The spectral analysis and detection optics were identical for both experiments. Upon collection of the Rayleigh scattered light by the $0.55-\mathrm{mm}$ diameter multimode fiber, it was routed to a separate area, shown schematically in Fig. 15. The light exiting the fiber was collimated by an 80 -mm focal length $f / 1.6$ lens (L6) and was directed through the planar mirror Fabry-Perot interferometer. The FPI had 70-mm diameter mirrors with 80\% reflectivity, $8.7 \mathrm{GHz}$ free spectral range, and reflective finesse of approximately 15. The Fabry-Perot is an extremely sensitive instrument; even the smallest vibrations or temperature changes can cause the mirrors to drift out of parallel alignment, resulting in increased uncertainty. Therefore, a stabilization system was utilized to maintain parallelism of the mirrors. Between Rayleigh scattering measurements, a mirror and diffuser (shown in dashed gray lines in Fig. 13) were placed in the beam path by linear actuators to direct some of the incident laser beam into the optical fiber and through the FPI. A similar mirror and diffuser system were used in the SHJAR testing, however the details are not shown in Fig. 14. A set of reflecting prisms mounted on a linear actuator (Fig. 15) were positioned in the optical path at the output of the interferometer to direct the light from three regions of the interferometer mirrors to a video camera. Live video of the three fringe images was used in a feedback control loop to adjust the mirror positions by controlling the voltage applied to piezoelectric transducers on the mirror mounts until the three fringes were equal in diameter indicating that the mirrors are equally spaced at all three locations. This system was also used to set the fringe diameter of the incident reference light to $13-\mathrm{mm}$ for the electrically-heated jet experiment, and 14.4-mm for the SHJAR test.

When flow measurements were acquired, the prisms, mirror, and diffuser were removed from the optical path and the Rayleigh scattered light exiting the FPI was focused by a fringe forming lens (L7) having an effective focal length of approximately $2700-\mathrm{mm}$, which provided a 18.6- $\mathrm{mm}$ diameter image of the fiber face. Light from circular and annular sections of the image were directed toward PMTs by a concentric elliptical mirror system, which was designed and fabricated specifically for this application. A photograph of the 'image dissector' is shown in the upper left corner of Fig. 15. The mirrors were machined from 6061 aluminum and the $12^{\circ}$ elliptical surfaces were diamond polished to a mirror finish with about $90 \%$ reflectivity. The mirror diameters, from innermost to outermost, are 5$\mathrm{mm}, 12-\mathrm{mm}, 16-\mathrm{mm}$, and 25-mm. Each mirror sends the respective portion of the fringe image toward PMTs 1, 2, 3, and 4, as shown previously in Fig. 6; the fringe regions are also illustrated near the corresponding PMT in Fig. 15.

The PMTs were operated in the photon counting mode to acquire fringe intensity data. Each PMT was supplied with $1200 \mathrm{~V}$ and the varying amplitude photoelectron pulses from the five PMTs were amplified with a gain of 5 and were counted by Stanford Research model SR400 photon counters, which output 6 ns wide NIM level (-700 $\mathrm{mV}$ ) pulses for each incoming photoelectron pulse having an amplitude exceeding the threshold level. Typical threshold levels ranged from $-25 \mathrm{mV}$ to $-50 \mathrm{mV}$ for the five PMTs. Canberra model 2126 constant fraction

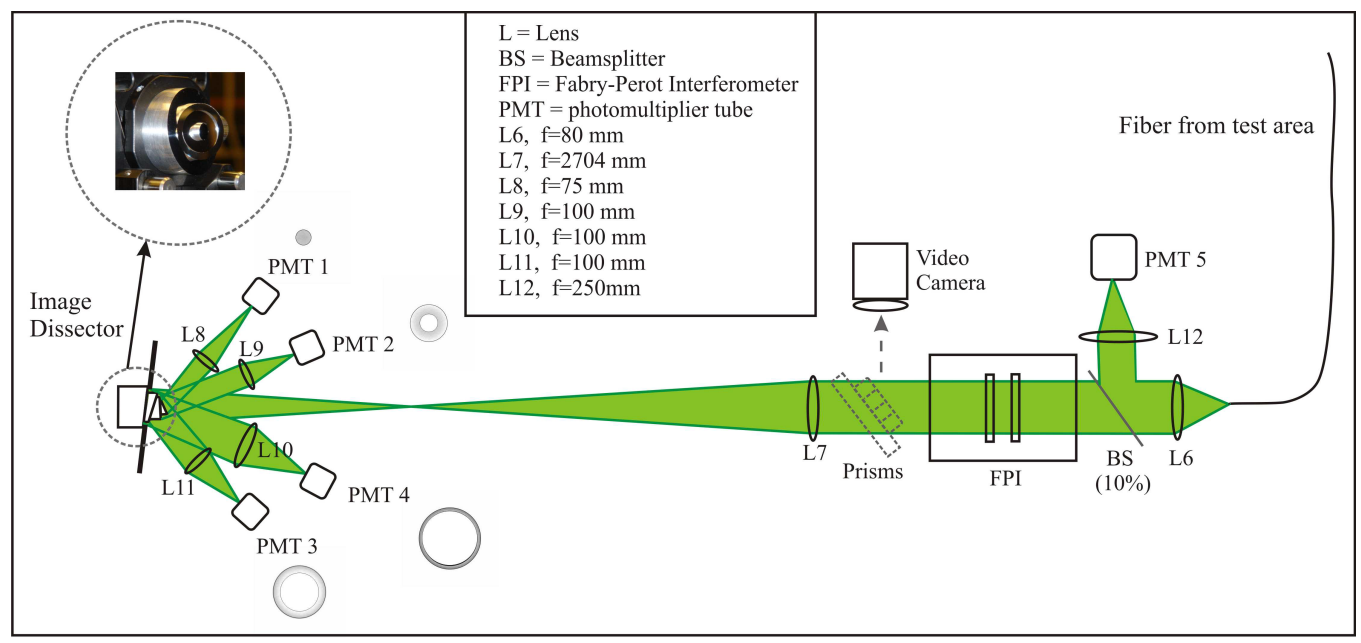

Figure 15. Schematic of spectral analysis and detection optics. 
discriminators converted the NIM level pulses to $8 \mathrm{~ns}$ wide TTL level $(+2.5 \mathrm{~V})$ pulses that were counted by two National Instruments model 6602 counter-timer boards. Typical photoelectron count rates for this work were on the order of $1 \mathrm{MHz}$. A PC-based data acquisition system utilizing National Instruments data acquisition hardware and LabVIEW software was used to record the signals from the PMTs. Each signal channel was digitized for either 60 seconds at 1,10 , or $16 \mathrm{kHz}$ sampling rate or 10 seconds at $32 \mathrm{kHz}$ rate, resulting in $60,000,600,000,960,000$, or 320,000 samples per channel. The intensity information from the Fabry-Perot fringe sampling was used to obtain temperature and velocity measurements. The density measurement was much simpler, requiring only an overall intensity measurement. A beamsplitter located just in front of the FPI input (Fig. 15) directed approximately 10\% of the incoming Rayleigh scattered light to a lens (L12), which focused the light at PMT 5 for the density measurement.

\section{Data Analysis}

\section{A. Experiment calibration and data analysis method}

The technique was calibrated over a range of steady flow operating points in each flow system. The electricallyheated jet was operated over a static temperature range of $298-765 \mathrm{~K}$ and velocity range of $14-115 \mathrm{~m} / \mathrm{s}$ and the SHJAR was operated over a temperature range of $239-630 \mathrm{~K}$ and velocity range of $167-300 \mathrm{~m} / \mathrm{s}$. The photoelectron counts recorded by PMT 5 are linearly related to density. The photon count measurement from PMT 5 provides an independent yet simultaneous measurement of density along with the velocity and temperature measurements from spectroscopic analysis. The axial velocity component and temperature were measured by analyzing the light from the circular and annular regions of the interference fringe pattern at the output of the FPI. The model of the fringe pattern (Eq. (13)), was used in the MLE analysis to estimate unknown parameters from the intensity data, such as velocity and temperature, or unknown optical system parameters. For calibration, mean temperature, velocity, density, and pressure measured by physical probes or calculated from the isentropic flow and ideal gas relations were used as known values in the model function. Several unknown parameters were evaluated by MLE analysis of the mean photon count data at the calibration points. These unknown parameters included system detection efficiencies, effective finesse of the FPI, and fringe forming lens focal length. Once determined, these parameters were input into the model function for use in MLE analysis of the PMT counts for temperature and velocity measurements.

Using the linear relation between PMT 5 photon counts and density, and MLE analysis of the photon counts from PMTs 1-4, time histories of instantaneous density, temperature, and velocity were evaluated. Although a constant static pressure equivalent to the ambient pressure is probably a valid assumption in these subsonic free jet experiments, the pressure was assumed to be unknown. The density measured by PMT 5 and the temperature estimate from MLE analysis of PMT 1-4 photon counts were used to determine the local pressure using the ideal gas law to demonstrate that this measurement technique is applicable in flows where the pressure is unknown and the ideal gas law is valid.

\section{B. Power spectral calculations}

The mean velocity, temperature, and density were calculated and subtracted from their respective time history records to provide zero-mean records, which were used to calculate the power spectral densities of the property fluctuations. Because of the shot noise in the PMT signals, it was necessary to use relatively long data records and calculate power spectra using a technique known as the Welch method of modified periodograms ${ }^{27}$. Due to extensive processing time of the Rayleigh data (approximately 1000 samples/minute), only the first 10.24 and 5.12 seconds were analyzed for the $16 \mathrm{kHz}$ and $32 \mathrm{kHz}$ data rates, respectively, in the electrically-heated jet experiment, and the first 7.98 seconds for the $10 \mathrm{kHz}$ data in the SHJAR experiment. In the Welch method, a data record sampled at a rate $f_{s}=10,16$ or, $32 \mathrm{kHz}$ was subdivided into smaller records of length $L=1024,2048$, or 4096 samples, which were overlapped by $50 \%$. The modified periodograms of each sub-record were calculated using a Welch data window. These individual periodograms were then averaged to obtain the estimate of the power spectrum. The number of individual periodograms used to calculate the averaged power spectrum depends on the length of the record used. The 5.12 second data records resulted in 78 overlapped segments, the 10.24 second data records resulted in 158 overlapped segments, and the 7.98 second data records resulted in 154 overlapped segments. The frequency resolution of the resulting spectra is $f_{s} / L=7.8125 \mathrm{~Hz}$ for the 16 and $32 \mathrm{kHz}$ data and $9.7656 \mathrm{~Hz}$ for the $10 \mathrm{kHz}$ data. The hotwire data was sampled at $32 \mathrm{kHz}$, but the coldwire data was sampled at only $1 \mathrm{kHz}$ due to the response limit of the system. Overlapping the segments by $50 \%$ provided a near maximum reduction in the variance in the spectral estimate ${ }^{27}$. The resulting power spectra from the Rayleigh scattering measurements provide 
fluctuation information up to half of the sampling frequency $(5,8$, or $16 \mathrm{kHz})$, and the sum of all points in the spectra is equivalent to the mean square fluctuations.

A common numerical processing method used to eliminate noise from power spectra involves simply subtracting the noise floor from the spectrum. Shot noise is broadband noise that contributes equally over all frequencies; therefore it causes a constant offset in the spectrum. If the spectrum flattens out at high frequencies due to the lack of significant fluctuations, then the average value of this noise floor can be estimated and subtracted from every point in the spectrum, eliminating the broadband shot noise contribution. This procedure was used to eliminate shot noise in the data presented in this paper; the noise floor was estimated from the last $1000 \mathrm{~Hz}$ of the power spectrum.

\section{Results and Discussion}

\section{A. Data from the electrically-heated jet experiment}

The electrically-heated jet was operated at conditions which provided moderate temperature and velocity levels where resistance probes were applicable for measurement comparisons. The Rayleigh probe volume and the hotwire and coldwire probes were scanned horizontally across the centerline of the electrically-heated jet at an axial station of $x / D=2$. Scans of the Rayleigh probe volume were performed at ideally expanded jet conditions of $420 \mathrm{~K}$ and 38 $\mathrm{m} / \mathrm{s}$ (case 1) and $420 \mathrm{~K}$ and $110 \mathrm{~m} / \mathrm{s}$ (case 2). Due to the fragility of the coldwire probe it could only be used to provide temperature fluctuation measurements in the low velocity flow. The hotwire probe also had physical limitations leading to the inability to make measurements in heated flows. Therefore, hotwire measurements were performed in cold flows with maximum velocities similar to the heated flow cases. The radial scans provided measurements in the jet core as well as in the turbulent mixing layer formed between hot and cold air streams. The mean temperature $(T)$ and velocity $\left(u_{k}\right)$ values for the Rayleigh measurements were calculated from the time-history data, and the rms temperature $\left(T^{\prime}\right)$ and velocity $\left(u_{k}{ }^{\prime}\right)$ fluctuations were calculated from the power spectra by taking the square root of the sum of all points in the spectra after the noise floor had been subtracted.

Figure 16 shows the radial profile across the jet flow at $x / D=2$ of the mean temperature, and Fig. 17 shows the radial profile of rms temperature fluctuations normalized by the difference between the jet exit temperature and the ambient temperature $\left(T_{i d}-T_{a m b}\right)$, calculated from the 1,16 , and $32 \mathrm{kHz}$ Rayleigh measurements. The Rayleigh measurements are compared with thermocouple and $1 \mathrm{kHz}$ coldwire temperature measurements in these figures. The mean temperatures from the Rayleigh technique compare well with the thermocouple measurements which were acquired in the flow just prior to the Rayleigh data acquisition, with the greatest deviation being less than $2 \%$. Deviation between the Rayleigh and coldwire mean temperatures is slightly greater; however the coldwire measurements were acquired on a different day and the jet conditions may not have been exactly duplicated. The 1 $\mathrm{kHz}$ Rayleigh temperature fluctuations match the $1 \mathrm{kHz}$ coldwire measurements very well. The largest deviation between the two measurements is approximately $1.5 \%$ and occurs in the jet core. The coldwire measurements provide very accurate turbulence levels for fluctuations up to $500 \mathrm{~Hz}$; however, significant temperature fluctuations most likely exist beyond $500 \mathrm{~Hz}$. Since the $1 \mathrm{kHz}$ coldwire and Rayleigh measurements do not account for higher frequency fluctuations, the resulting turbulence levels from these data sets are lower than the true temperature fluctuations over all frequencies. The peak temperature fluctuations calculated from the direct Rayleigh temperature measurements range from $16 \%$ to $19 \%$, agreeing with the rule of thumb that shear layer fluctuation levels are typically $15 \%$ to $20 \%$. Greater error is expected in the lower amplitude fluctuation measurements due to the nonzero temperature fluctuation amplitude measurement lower bound, which was determined to be $5-11.5 \mathrm{~K}$ for a 32 $\mathrm{kHz}$ data rate by the numerical simulation analysis discussed in section II.B.

Figure 18 shows a radial profile of mean velocity at $x / D=2$, and Fig. 19 shows a radial profile of rms velocity fluctuations normalized by the ideally expanded exit velocity $\left(U_{i d}\right)$. This data was calculated from 16 and $32 \mathrm{kHz}$ Rayleigh measurements and are compared with $32 \mathrm{kHz}$ hotwire velocity measurements acquired in an unheated flow at the same maximum velocity as the heated flow of case 2 . The asymmetry of the velocity profiles is caused by flow disturbance from the kanthal heater coils in the jet plenum. The mean velocities from the Rayleigh measurements show some deviation from the hotwire measurements at a few points in the radial profile. The Rayleigh velocity measurements in the low velocity region of the co-flow tend to be biased towards lower values than those measured by the hotwire probe by as much as $5-10 \mathrm{~m} / \mathrm{s}$. The agreement between the measurements gets better as velocity increases; the Rayleigh measurements agree with the hotwire measurements within $5 \mathrm{~m} / \mathrm{s}$ throughout most of the profile. The laser used for these measurements was not frequency stabilized; there was inherent drift in the laser frequency over time. Previous analysis ${ }^{26}$ quantified the amount of velocity error induced by frequency drift of this particular laser system. The expected laser frequency drift over the time period of the Rayleigh data acquisition leads to potential velocity bias error on the order of $4 \mathrm{~m} / \mathrm{s}$. Error due to the accuracy with which the reference fringe radius is set by the Fabry-Perot stabilization system results in additional bias error of up 
to $2 \mathrm{~m} / \mathrm{s}$. The hotwire measurements provide shear layer fluctuation levels that are in line with the $15 \%$ to $20 \%$ rule of thumb; however the Rayleigh measurements indicate fluctuation levels $2 \%$ to $3.5 \%$ greater than the hotwire data at the peaks and as much as $12 \%$ greater in other regions of the profile. The discrepancies may be due to erroneous velocity fluctuations related to instability of the Rayleigh laser frequency and a non-zero fluctuation amplitude measurement lower bound as discussed in the uncertainty analysis.

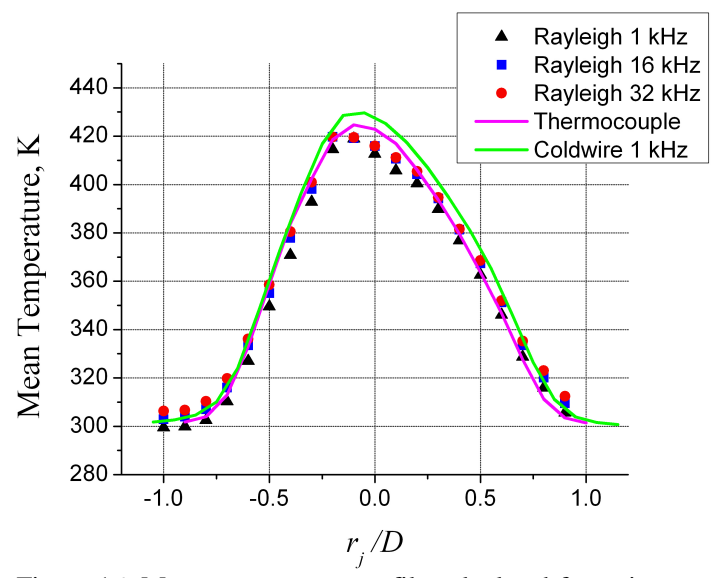

Figure 16. Mean temperature profile calculated from timeresolved Rayleigh and coldwire data and measured by a thermocouple for a flow with ideally expanded temperature and velocity of $T_{i d}=420 \mathrm{~K}$ and $U_{i d}=38 \mathrm{~m} / \mathrm{s}$.

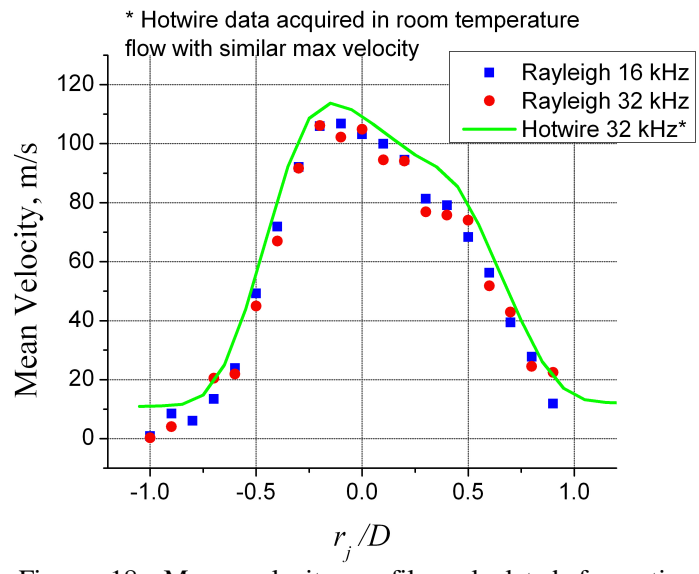

Figure 18. Mean velocity profile calculated from timeresolved Rayleigh data and measured by a pitot tube in a flow with ideally expanded temperature and velocity of $T_{i d}=420$ $\mathrm{K}$ and $U_{i d}=110 \mathrm{~m} / \mathrm{s}$, and hotwire measurements in a room temperature flow with similar maximum velocity.

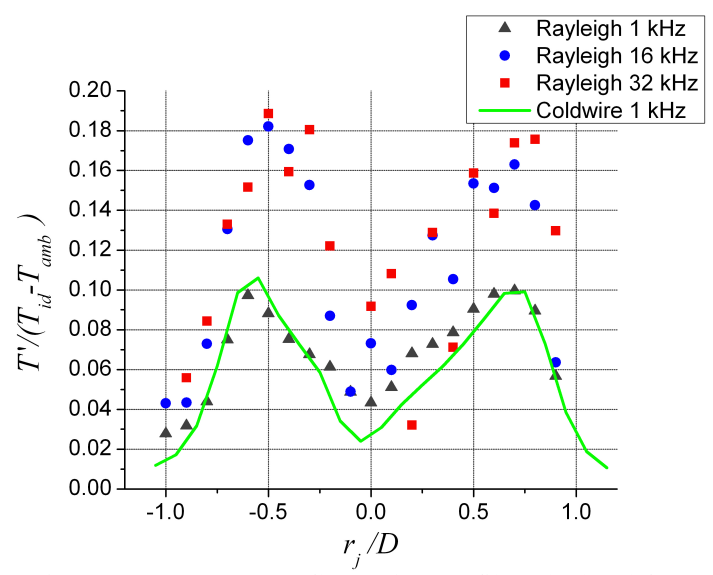

Figure 17. Temperature fluctuation profile calculated from Rayleigh and coldwire measurements in a flow with ideally expanded temperature and velocity of $T_{i d}=420 \mathrm{~K}$ and $U_{i d}=$ $38 \mathrm{~m} / \mathrm{s}$.

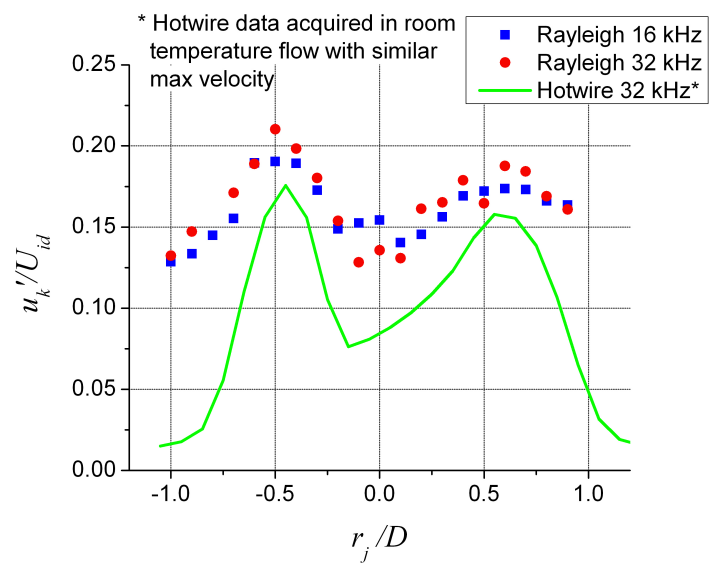

Figure 19. Velocity fluctuation profile calculated from Rayleigh measurements in a flow with ideally expanded temperature and velocity of $T_{i d}=420 \mathrm{~K}$ and $U_{i d}=110 \mathrm{~m} / \mathrm{s}$ and hotwire measurements in a room temperature flow with similar maximum velocity.

\section{B. Data from the SHJAR}

The SHJAR was operated at ideally expanded conditions of $U_{i d}=167 \mathrm{~m} / \mathrm{s}$ and $T_{i d}=496 \mathrm{~K}(\mathrm{Ma}=0.5$, TR $=$ $1.765)$ while the Rayleigh probe volume was scanned horizontally from the jet centerline $\left(r_{j} / D=0\right)$ outward through the shear layer to a point in the co-flow $\left(r_{j} / D=1.1\right)$ at an axial location of $x / D=3$. Hotwire and PIV data were acquired in the same facility several years prior to this test and are included here for comparison with the Rayleigh measurements. Figure 20 shows the mean velocity normalized by the ideally expanded velocity as a function of normalized radial position $\left(r_{j} / D\right)$ for Rayleigh data acquired at $10 \mathrm{kHz}$, hotwire data acquired in a cold flow with the same Mach number of 0.5 at a sampling rate of $10 \mathrm{kHz}$, and PIV data acquired at the same hot jet conditions as the Rayleigh data at a sampling rate of $10 \mathrm{~Hz}$. Minor differences in the mean velocities exist between the three measurements. The hotwire data was acquired in a cold flow and is expected to show some differences. The Rayleigh data shows a slight underestimation of the mean velocities, which may be partly due to velocity bias 
error associated with laser frequency drift. Figure 21 shows a radial profile of rms velocity fluctuations normalized by the ideally expanded velocity based on the three different measurement techniques. The PIV data acquired at the much lower sampling rate provides rms velocity fluctuation information via ensemble averaging of many snapshots; however it is not capable of providing power spectral information. PIV data that is temporally resolved at a rate of $10 \mathrm{kHz}$ has been acquired in this facility but is not available for these jet conditions. ${ }^{8}$. Although the hotwire data was acquired at $10 \mathrm{kHz}$, a $4 \mathrm{kHz}$ anti-aliasing filter was used which causes some loss in energy since the fluctuations associated with higher frequencies are filtered from the signal; although the reduction should be small for $x / D>1$ because there is not much signal strength at the higher frequencies. As expected, the hotwire data acquired in a cold flow indicates a broader potential core and lower fluctuation levels than the heated flow case. The rms velocity fluctuations from the Rayleigh technique were calculated from the power spectra by first subtracting the noise floor, summing all points in the corrected spectrum, and finally taking the square root of that sum. This method provides accurate rms fluctuation estimates in the regions on either side of the shear layer; however within the shear layer the noise floor subtraction technique removes energy that falls below the noise floor but actually is present, particularly in the higher frequency range. A plot of the power spectra at $r_{j} / D=0.44$ is shown in Fig. 22 to illustrate the spectra prior to subtraction of the noise floor (blue line) and after the noise floor has been subtracted (red line). The green line is the power spectrum for zero-turbulence synthetic Rayleigh data having mean temperature and velocity similar to the actual values at this location in the flow field generated using the numerical simulation discussed in section II.B. The simulated data provides an estimate of the expected noise floor due to Poisson noise. It is clearly evident that the level of the uncorrected spectrum (blue line) is approximately equal to the noise floor level predicted by the simulation (green line) over the last 1000 to $2000 \mathrm{~Hz}$ of the spectrum. If the total power level in a frequency bin is below the noise floor level, the contribution of the fluctuations in that frequency range will not be properly taken into account. The cross-hatched blue area indicates a prediction of how the spectrum may continue to fall off and the amount of energy lost due to the inability to accurately take these fluctuations into account. The power in the crosshatched area corresponds to a fluctuation level of $u_{k}{ }^{\prime} / U_{i d}=0.035$, which is the difference between the PIV and Rayleigh fluctuation estimates at $r_{j} / D=0.44$. Measurements outside of the shear layer typically do not encounter this problem since the strength of the fluctuations at higher frequencies is negligible. A problem does arise when the fluctuations fall below the noise floor over the entire spectrum, as is the case at $r_{j} / D=1.1$, because all fluctuation information is lost in the noise of the technique. Therefore, it is important to consider the expected fluctuation levels when implementing the Rayleigh technique; the technique works best in flows with high turbulence levels that significantly exceed the Poisson noise baseline level.

Figures 23 and 24 show radial profiles (one-half of the jet only) of mean and rms temperature, respectively, measured by the Rayleigh technique normalized by the difference between the ideally expanded and ambient temperatures $\left(T_{i d}-T_{a m b}\right)$ for the same jet conditions of $T_{i d}=500 \mathrm{~K}$ and $U_{i d}=167 \mathrm{~m} / \mathrm{s}$. Comparison temperature data is not yet available; however the peak rms fluctuations are expected to be between $15 \%$ and $20 \%$. The fluctuations measured by the Rayleigh technique are slightly higher $(21 \%)$. The fluctuations at the jet centerline and in the surrounding co-flow are probably less than $1 \%$ which is in the noise level of this technique.

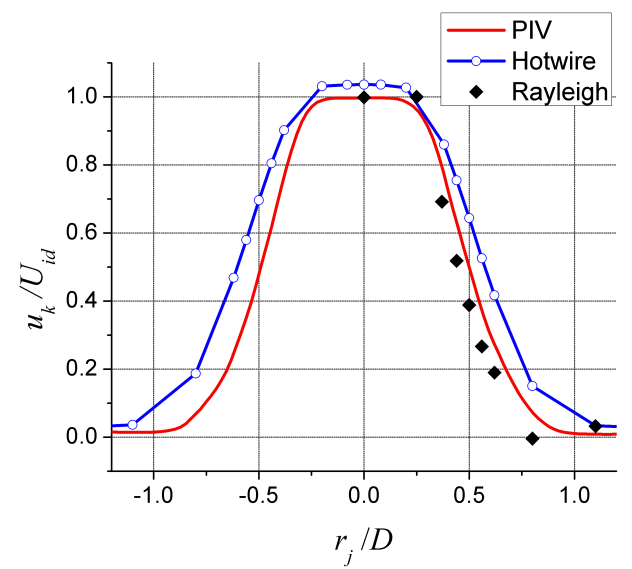

Figure 20. Mean velocity normalized by the ideally expanded velocity as a function of $r_{j} / D$ at an axial location of $x / D=3$ and jet exit conditions of $T_{i d}=500 \mathrm{~K}$ and $U_{i d}=$ $167 \mathrm{~m} / \mathrm{s}$ in the SHJAR facility. Rayleigh measurements are compared with hotwire and PIV data.

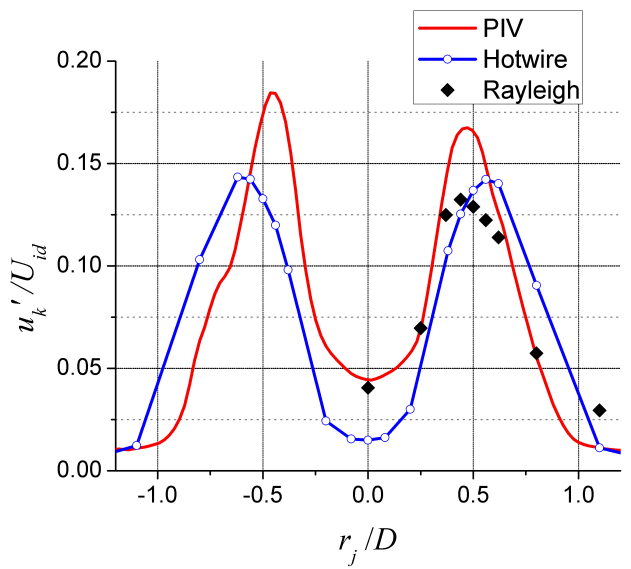

Figure 21. Velocity rms normalized by the ideally expanded velocity as a function of $r_{j} / D$ at an axial location of $x / D=3$ and jet exit conditions of $T_{i d}=500 \mathrm{~K}$ and $U_{i d}=167 \mathrm{~m} / \mathrm{s}$ in the SHJAR facility. Rayleigh measurements are compared with hotwire and PIV data. 


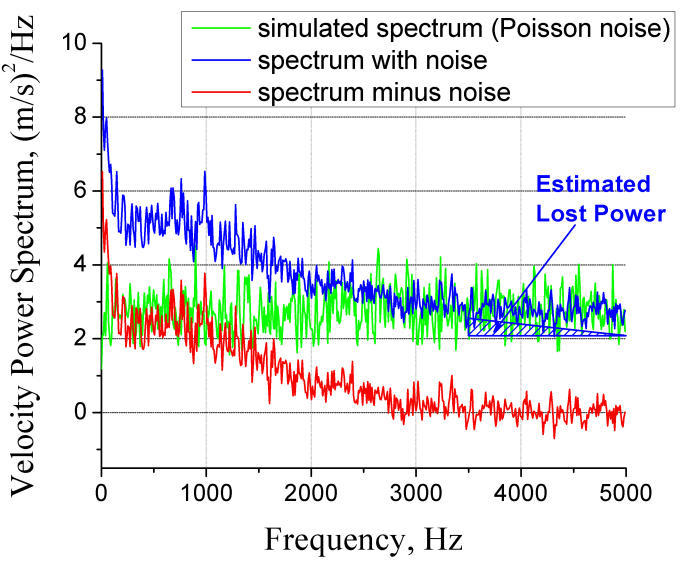

Figure 22. Velocity power spectra calculated from Rayleigh data acquired at $x / D=3, r_{j} / D=0.44$ in the SHJAR with nozzle exit conditions of $T_{i d}=500 \mathrm{~K}$ and $U_{i d}=167 \mathrm{~m} / \mathrm{s}$, and a spectrum calculated from zero-turbulence synthetic data with mean temperature and velocity similar to the actual values at $x / D=3, r_{j} / D=0.44\left(T=398 \mathrm{~K}, u_{k}=81.6 \mathrm{~m} / \mathrm{s}\right)$.

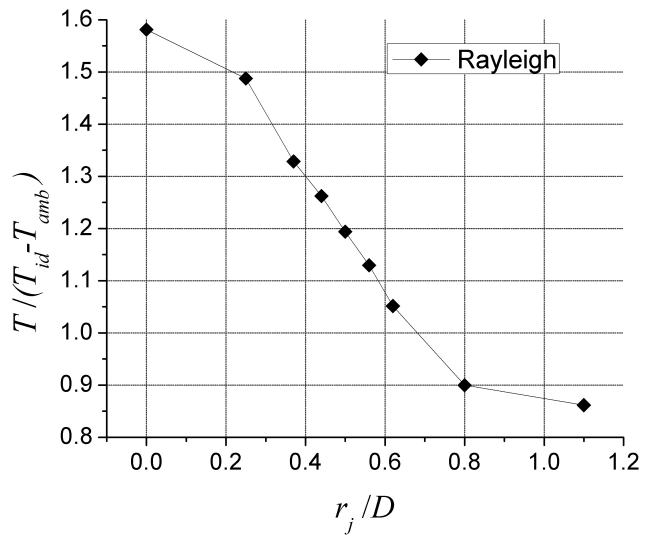

Figure 23. Mean temperature normalized by the ideally expanded temperature as a function of $r_{j} / D$ at an axial location of $x / D=3$ and jet exit conditions of $T_{i d}=500 \mathrm{~K}$ and $U_{i d}=167 \mathrm{~m} / \mathrm{s}$ in the SHJAR facility.

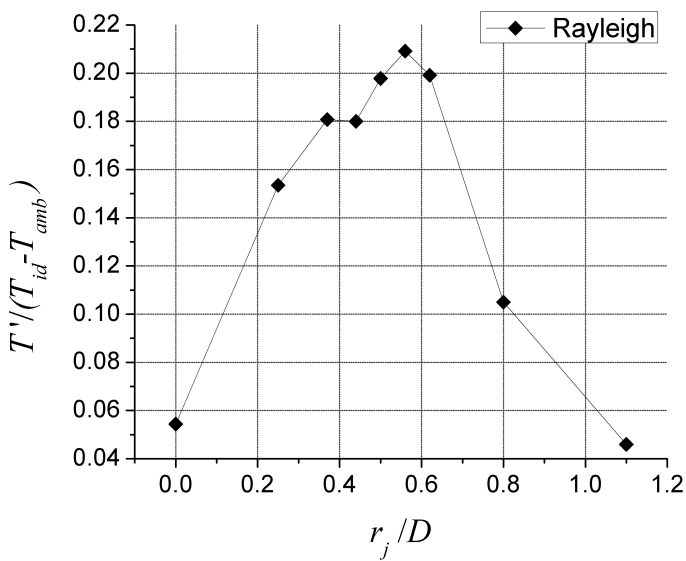

Figure 24. Temperature rms normalized by the difference between the ideally expanded temperature and the ambient temperature as a function of $r_{j} / D$ at an axial location of $x / D=3$ and jet exit conditions of $T_{i d}=500 \mathrm{~K}$ and $U_{i d}=167 \mathrm{~m} / \mathrm{s}$ in the SHJAR facility.

\section{Conclusion}

A technique for obtaining time-resolved gas velocity, temperature, and density measurements using molecular Rayleigh scattering was described. Density was determined from an overall intensity measurement of the scattered light, while temperature and velocity were determined by analyzing the scattered light with a Fabry-Perot interferometer. The signals from five photomultiplier tubes were simultaneously recorded using photon counting at sampling rates up to $32 \mathrm{kHz}$. An uncertainty analysis was presented that demonstrated uncertainties in instantaneous temperature and velocity measurements of about $25 \%$ and $37 \mathrm{~m} / \mathrm{s}$, respectively, for a $32 \mathrm{kHz}$ sampling rate and experiment parameters similar to those of the electrically-heated jet experiment. Other statistical quantities, such as mean square fluctuations and power spectra, can be obtained with higher accuracy as demonstrated by the numerical simulation analysis. The lowest measurable temperature and velocity fluctuation amplitudes were predicted to be 5 $11 \mathrm{~K}$ and $2-5 \mathrm{~m} / \mathrm{s}$, respectively, for a $32 \mathrm{kHz}$ sampling rate. The Rayleigh technique was demonstrated in two hot jet facilities: an electrically-heated jet with a 10-mm diameter nozzle and the Small Hot Jet Acoustic Rig (SHJAR) with a 50.8-mm diameter nozzle in NASA GRC's AeroAcoustic Propulstion Laboratory. The complete set of data acquired in the SHJAR will be analyzed and presented in a later publication to provide an experimental database for validation of computational codes. 


\section{Acknowledgments}

The authors would like to thank Dennis Eck and Ray Loew for their support in the setup and operation of the SHJAR facility and also Pete Eichele for his assistance in the setup and installation of the Rayleigh scattering system. We would also like to thank Dr. Mark Wernet and Dr. James Bridges for providing the PIV and hotwire data that was presented, and Professor Tenti for providing the 6-moment Rayleigh spectrum calculation code.

\section{References}

${ }^{1}$ Eckbreth, A. C., Laser Diagnostics for Combustion Temperature and Species, Gordon and Breach Science Publishers SA, Amsterdam, 1996, pp.209-451.

${ }^{2}$ Cummings, E. B., "Laser-Induced Thermal Acoustics," Ph.D. Dissertation, Department of Engineering and Applied Science, California Institute of Technology, Pasadena, CA, 1995.

${ }^{3}$ Hart, R. C., Balla, R. J., and Herring, G. C., "Nonresonant Referenced Laser-Induced Acoustics Thermometry in Air," Appl Optics, Vol. 38, No. 3, 1999, pp. 577-584.

${ }^{4}$ Boguszko, M., and Elliott, G. S., "On the Use of Filtered Rayleigh Scattering for Measurements in Compressible Flows and Thermal Fields," Exp. Fluids, Vol. 38, No. 1, 2005, pp. 33-49.

${ }^{5}$ Bonnet, J. P., Grésillon, D., Cabrit, B., and Frolov, V., "Collective Light Scattering as Non-Particle Laser Velocimetry," Meas. Sci. Technol., Vol. 6, No. 6, 1995, pp. 620-636.

${ }^{6}$ Koochesfahani, M. M., "Molecular Tagging Velocimetry (MTV): Progress and Applications,” AIAA-99-3786, 1999.

${ }^{7}$ Tropea, C., "Laser Doppler Anemometry: Recent Developments and Future Challenges," Meas. Sci. Technol., Vol. 6, No. 6, 1995, pp. 605-619.

${ }^{8}$ Wernet, M. P., "Temporally Resolved PIV for Space-Time Correlations in Both Cold and Hot Jet Flows," Meas Sci Technol, Vol. 18, No. 5, 2007, pp. 1387-1403.

${ }^{9}$ Samimy, M., and Wernet, M. P., "Review of Planar Multiple-Component Velocimetry in High-Speed Flows," AIAA Journal, Vol. 38, No. 4, 2007, pp. 553-574.

${ }^{10}$ Lock, J. A., Seasholtz, R. G., and John, W. T., "Rayleigh-Brillouin Scattering to Determine One-Dimensional Temperature and Number Density Profiles of a Gas Flow Field," Appl Optics, Vol. 31, No. 15, 1992, pp. 2839-2848.

${ }^{11}$ Seasholtz, R. G., and Greer, L. C., "Rayleigh scattering diagnostic for measurement of temperature and velocity in harsh environments," AIAA-98-0206, 1998.

${ }^{12}$ Panda, J., and Seasholtz, R. G., "Velocity and Temperature Measurement in Supersonic Free Jets Using Spectrally Resolved Rayleigh Scattering," AIAA-99-0296, 1999.

${ }^{13}$ Mielke, A. F., Seasholtz, R. G., Elam, K.A., and Panda, J., “Time-average Measurement of Velocity, Density, Temperature, and Turbulence Velocity Fluctuations Using Rayleigh and Mie Scattering," Exp Fluids, Vol. 39, No. 2, 2005, 441-454.

${ }^{14}$ Seasholtz, R. G., and Panda, J., "Rayleigh Scattering Diagnostic for Dynamic Measurement of Velocity and Temperature," AIAA-99-0641, 1999.

${ }^{15}$ Seasholtz, R. G., Panda, J., and Elam, K. A., "Rayleigh Scattering Diagnostic for Measurement of Velocity and Density Fluctuation Spectra," AIAA-2002-0827, 2002.

${ }^{16}$ Mielke, A. F., Elam, K. A., and Sung, C. J., "Development of a Rayleigh Scattering Diagnostic for Time-Resolved Gas Phase Flow Velocity, Temperature, and Density Measurements in Aerodynamic Test Facilities," $22^{\text {nd }}$ International Congress on Instrumentation in Aerospace Simulation Facilities, Vol. 1., IEEE, Washington, D.C., 2007, pp. 1-15.

${ }^{17}$ Panda, J., Seasholtz, R. G., Elam, K. A., Mielke, A. F., Eck, D. G., "Effect of Heating on Turbulent Density Fluctuations and Noise Generation from High Speed Jets," AIAA-2004-3016, 2004.

${ }^{18}$ Tenti, G., Boley, C., D., and Desai, R. C., "On the Kinetic Model Description of Rayleigh-Brillouin Scattering from Molecular Gases,” Can J Phys, Vol. 52, No. 4, 1974, pp. 285-290.

${ }^{19}$ Yip, S., and Nelkin, M., "Application of a Kinetic Model to Time-Dependent Density Correlations in Fluids," Phys Rev, Vol. 135, No. 5A, 1964, pp. A1241-A1247.

${ }^{20}$ Sandoval, R. P., and Armstrong, R. L., "Rayleigh-Brillouin Spectra in Molecular Nitrogen," Phys Rev A, Vol. 13, No. 2, 1976, pp. 752-757.

${ }^{21}$ Landau, L., and Placzek, G., "Structure of the Undisplaced Scattering Line," Physik Z. Sowjetunion, Vol. 5, 1934, p. 172.

${ }^{22}$ Clark, N. A., "Inelastic Light Scattering from Density Fluctuations in Dilute Gases: The Kinetic Hydrodynamic Transition in a Monoatomic Gas," Phys. Rev. A, Vol. 12, No. 1, 1975, pp. 232-244.

${ }^{23}$ Boley, C. D., Desai, R. C., and Tenti, G., "Kinetic Models and Brillouin Scattering in a Molecular Gas," Can J Phys, Vol. 50, No. 18, 1972, pp. 2158-2173.

${ }^{24}$ Vaughan, J. M., The Fabry Perot Interferometer, History, Theory, Practice, and Applications, Adam Hilger, Philadelphia, 1989, pp. 89-134.

${ }^{25}$ Whalen, A. D., Detection of Signals in Noise, Academic Press, New York, 1971, pp. 327-332.

${ }^{26}$ Mielke, A. F., "Development of a Molecular Rayleigh Scattering Diagnostic for Simultaneous Time-Resolved Measurement of Temperature, Velocity, and Density," Ph.D. Dissertation, Department of Mechanical and Aerospace Engineering, Case Western Reserve Univ., Cleveland, OH, 2008.

${ }^{27}$ Welch, P. D., "The Use of Fast Fourier Transform for the Estimation of Power Spectra: A Method Based on Time Averaging Over, Short Modified Periodograms," IEEE Trans on Audio and Electroacoustics, Vol. AU-15, No.2, 1967, pp. 70-73. 